\title{
Many-body applications of the stochastic limit: a review
}

\author{
F. Bagarello \\ Dipartimento di Metodi e Modelli Matematici, Facoltà di Ingegneria, Università di Palermo, \\ Viale delle Scienze, I-90128 Palermo, Italy \\ e-mail: bagarell@unipa.it \\ home page: www.unipa.it/ bagarell
}

\begin{abstract}
We review some applications of the perturbative technique known as the stochastic limit approach to the analysis of the following many-body problems: the fractional quantum Hall effect, the relations between the Hepp-Lieb and the Alli-Sewell models (as possible models of interaction between matter and radiation), and the open BCS model of low temperature superconductivity.
\end{abstract}

Keywords: Stochastic limit. BCS model. Quantum Hall effect. Laser models.

PACS Numbers: 02.90.+p, 03.65.Db 


\section{Introduction}

In this paper we review with a certain care the main results concerning three applications of the so-called stochastic limit approach (SLA), see [1] for the main definitions and some physical applications and [2] for some rigorous mathematical results, to three problems in quantum mechanics for systems with infinite degrees of freedom. In particular we devote Section II to the discussion of the fractional quantum Hall effect, [3]. In Section III we discuss many relations between different models of matter interacting with radiation: the Hepp-Lieb and the DickeHaken-Lax hamiltonian models, and the Alli-Sewell dissipative counterpart, [4]. In Section IV, we discuss the open BCS model of superconductivity as originally proposed by Martin and Buffet, [5], and some quite recent results related to that, [6, 7]. For reader's convenience, we also add a brief Appendix where some crucial facts on the SLA are listed.

\section{The fractional quantum Hall effect}

The Hamiltonian for the quantum Hall effect (QHE) is, see for instance reference [8],

$$
H^{(N)}=H_{0}^{(N)}+\lambda\left(H_{c}^{(N)}+H_{B}^{(N)}\right)
$$

where $H_{0}^{(N)}$ is the Hamiltonian for the free $N$ electrons, $H_{c}^{(N)}$ is the Coulomb interaction:

$$
H_{c}^{(N)}=\frac{1}{2} \sum_{i \neq j}^{N} \frac{e^{2}}{\left|\underline{r}_{i}-\underline{r}_{j}\right|}
$$

and $H_{B}^{(N)}$ is the interaction of the charges with the positive uniform background. A huge literature exists concerning the QHE. We suggest here references [9] and [10].

In this section we consider a model defined by an Hamiltonian

$$
H=H_{0}^{(N)}+H_{0, R}+\lambda H_{e b}=H_{0}+\lambda H_{e b}
$$

which is obtained from the Hamiltonian (2.1) by introducing the following approximations:

(1) the Coulomb background-background interaction is replaced by the free bosons Hamiltonian $H_{0, R}$ given by

$$
H_{0, R}=\int \omega(\underline{k}) b^{+}(\underline{k}) b(\underline{k}) d k
$$

where $\omega(\underline{k})$ is the dispersion for the free background and $b^{+}(\underline{k})$ and $b(\underline{k})$ are bosonic operators satisfying the canonical comutation relations:

$$
\left[b(\underline{k}), b^{+}\left(\underline{k}^{\prime}\right)\right]=\delta\left(\underline{k}-\underline{k}^{\prime}\right) \quad\left[b(\underline{k}), b\left(\underline{k}^{\prime}\right)\right]=\left[b^{+}(\underline{k}), b^{+}\left(\underline{k}^{\prime}\right)\right]=0
$$


(2) the Coulomb electron-electron and electron-background interaction is replaced by the Fröhlich Hamiltonian $H_{e b}$, [11], which is only quadratic rather than quartic in the fermionic operators:

$$
H_{e b}=\int \psi^{\dagger}(\underline{r}) \psi(\underline{r}) \tilde{F}\left(\underline{r}-\underline{r}^{\prime}\right) \phi\left(\underline{r}^{\prime}\right) d r d r^{\prime}
$$

where $\psi(\underline{r})$ and $\phi\left(\underline{r}^{\prime}\right)$ are respectively the electron and the bosonic fields, while $\tilde{F}$ is a form factor. Expanding $\phi(\underline{r})$ in plane waves, $\psi(\underline{r})$ in terms of the eigenstates $\psi_{\alpha}(\underline{r})$ of the single electron hamiltonian, see (2.14) below, introducing the form factors

$$
g_{\alpha \beta}(\underline{k}):=\frac{1}{\sqrt{(2 \pi)^{3}}} \frac{\hat{V}_{\alpha \beta}(\underline{k})}{\sqrt{2 \omega(\underline{k})}}
$$

where

$$
\hat{V}_{\alpha \beta^{\prime}}(\underline{k}):=\int \overline{\psi_{\alpha}(\underline{r})} e^{i \underline{k} \cdot \underline{r}} \psi_{\beta^{\prime}}(\underline{r}) d r
$$

and taking $\tilde{F}(\underline{r})=e^{2} \delta(\underline{r})$, [11], we can write

$$
H_{e b}=e^{2} \sum_{\alpha \beta} a_{\alpha}^{+} a_{\beta}\left(b\left(g_{\alpha \beta}\right)+b^{+}\left(\overline{g_{\beta \alpha}}\right)\right)
$$

which is quadratic in the fermionic operators $a_{\alpha}, a_{\alpha}^{+}$,

$$
\left\{a_{\alpha}, a_{\beta}\right\}=\left\{a_{\alpha}^{+}, a_{\beta}^{+}\right\}=0 \quad\left\{a_{\alpha}, a_{\beta}^{+}\right\}=\delta_{\alpha \beta}
$$

Here we have introduced the smeared operators

$$
b\left(g_{\beta \alpha}\right)=\int d k b(\underline{k}) g_{\beta \alpha}(\underline{k}) .
$$

Notice that we are adopting here and in the following the simplifying notation for the quantum numbers $\alpha:=\left(n_{\alpha}, p_{\alpha}\right)$, see formula (2.14) below.

These are certainly strong approximations. However since from the Fröhlich Hamiltonian it is possible to recover a quartic interaction, [11], one can say that the Fröhlich Hamiltonian describes an effective electron-electron interaction which may mimic at least some aspects of the original Coulomb interaction. From this point of view it seems natural to conjecture that some dynamical phenomena deduced from this Hamiltonian might have implications in the study of the QHE. This conjecture is supported by our main result, given by formulae (2.69) and (2.70) where we deduce, directly from the dynamics, an obstruction to the presence of a non zero $x$-component of the current, which is quantized according to the values of a finite set of rational numbers. This is what we will call a fine tuning condition (FTC). 
Useless to say that the FTC strongly reminds the rational values of the filling factor for which the plateaux are observed in the real QHE. We will comment again this fact later.

In these notes we discuss a model of $N<\infty$ charged interacting particles concentrated around a two dimensional layer contained in the $(x, y)$-plane and subjected to a uniform electric field $\underline{E}=E \hat{j}$, along $y$, and to an uniform magnetic field $\underline{B}=B \hat{k}$ along $z$.

The Hamiltonian for the free $N$ electrons $H_{0}^{(N)}$, is the sum of $N$ contributions:

$$
H_{0}^{(N)}=\sum_{i=1}^{N} H_{0}(i)
$$

where $H_{0}(i)$ describes the minimal coupling of the $i$-th electrons with the field:

$$
H_{0}(i)=\frac{1}{2 m}\left(\underline{p}+\frac{e}{c} \underline{A}\left(r_{i}\right)\right)^{2}+e \underline{E} \cdot \underline{r}_{i}
$$

In the Landau gauge $\underline{A}=-B(y, 0,0)$, and requiring periodic boundary condition on $x$, $\psi\left(-L_{x} / 2, y\right)=\psi\left(L_{x} / 2, y\right)$, for almost all $y$, we find

$$
\psi_{n p}(\underline{r})=\frac{e^{i \frac{2 \pi p x}{L_{x}}}}{\sqrt{L_{x}}} \varphi_{n}\left(y-y_{0}^{(p)}\right) \quad \varepsilon_{n p}=\hbar \omega(n+1 / 2)-\frac{e E}{2 m \omega^{2}}\left(e E-\frac{4 \hbar \omega \pi p}{L_{x}}\right)
$$

where $\varphi_{n}$ is the $n$-th eigenstate of the one-dimensional harmonic oscillator, $\omega=\frac{e B}{m c}, y_{0}=$ $\frac{1}{m \omega^{2}}(\hbar k \omega-e E)$ and $k=\frac{2 \pi}{L_{x}} p$, where $p \in \mathbf{Z}$.

Equation (2.14) shows that the wave function $\psi_{n p}(\underline{r})$ factorizes in a $x$-dependent part, which is labelled by the quantum number $p$, and a part, only depending on $y$, which is labelled by both $n$ and $p$ due to the presence of $y_{0}^{(p)}$ in the argument of the function $\varphi_{n}$.

Of course the Hamiltonian $H_{0}^{(N)}$ in $(2.12)$ becames, in a second quantized form,

$$
H_{0}^{(N)}=\sum_{\alpha} \varepsilon_{\alpha} a_{\alpha}^{+} a_{\alpha}
$$

where the $\varepsilon_{\alpha}$ are the single electron energies, labeled by the pairs $\alpha=(n, p)$ as given in formula (2.14) and the $a_{\alpha}^{\sharp}$ are the fermionic operators satisfying (2.10).

\section{II.1 The stochastic limit of the model}

In this subsection we briefly outline how to apply the stochastic limit procedure to the model introduced above. The stochastic limit describes the dominating contribution to the dynamics in time scales of the order $t / \lambda^{2}$, where $\lambda$ is the coupling constant. 
The starting point is the Hamiltonian (2.3) together with the commutation relations (2.10), (2.5). Of course, the Fermi and the Bose operators commute among them. The interaction Hamiltonian $H_{e b}$ for this model is given by (2.9) and the free Hamiltonian $H_{0}$ is given by (2.3), (2.4) and (2.15).

The time evolution of $H_{e b}$, in the interaction picture is then

$$
H_{e b}(t)=e^{i H_{0} t} H_{e b} e^{-i H_{0} t}=e^{2} \sum_{\alpha \beta} a_{\alpha}^{+} a_{\beta}\left(b\left(g_{\alpha \beta} e^{-i t\left(\omega-\varepsilon_{\alpha \beta}\right)}\right)+b^{+}\left(\bar{g}_{\beta \alpha} e^{i t\left(\omega-\varepsilon_{\beta \alpha}\right)}\right)\right)
$$

where

$$
\varepsilon_{\alpha \beta}=\varepsilon_{\alpha}-\varepsilon_{\beta}
$$

Therefore the Schrödinger equation in the interaction representation is:

$$
\partial_{t} U_{t}^{(\lambda)}=-i \lambda H_{e b}(t) U_{t}^{(\lambda)}
$$

which becames, after the time rescaling $t \rightarrow t / \lambda^{2}$,

$$
\partial_{t} U_{t / \lambda^{2}}^{(\lambda)}=-\frac{i}{\lambda} H_{e b}\left(t / \lambda^{2}\right) U_{t / \lambda^{2}}^{(\lambda)}
$$

Its integral form is

$$
U_{t / \lambda^{2}}^{(\lambda)}=\mathbb{1}-\frac{i}{\lambda} \int_{0}^{t} H_{e b}\left(t^{\prime} / \lambda^{2}\right) U_{t^{\prime} / \lambda^{2}}^{(\lambda)} d t^{\prime}
$$

We see that the rescaled Hamiltonian

$$
\frac{1}{\lambda} H_{e b}\left(t / \lambda^{2}\right)=e^{2} \sum_{\alpha \beta} a_{\alpha}^{\dagger} a_{\beta} \frac{1}{\lambda} b\left(e^{\frac{-i t}{\lambda^{2}}}\left(\omega-\varepsilon_{\alpha \beta}\right) g_{\alpha \beta}\right)+\text { h.c. }
$$

depends on the rescaled fields

$$
b_{\alpha \beta, \lambda}(t)=\frac{1}{\lambda} b\left(e^{-i \frac{t}{\lambda^{2}}\left(\omega-\varepsilon_{\alpha \beta}\right)} g_{\alpha \beta}\right)
$$

The first statement of the stochastic golden rule, [1], is that the rescaled fields converge (in the sense of correlators) to a quantum white noise

$$
b_{\alpha \beta}(t)=\lim _{\lambda \rightarrow 0} \frac{1}{\lambda} b\left(g_{\alpha \beta} e^{-i \frac{t}{\lambda^{2}}\left(\omega-\varepsilon_{\alpha \beta}\right)}\right)
$$

characterized by the following commutation relations

$$
\begin{gathered}
{\left[b_{\alpha \beta}(t), b_{\alpha^{\prime} \beta^{\prime}}\left(t^{\prime}\right)\right]=\left[b_{\alpha \beta}^{+}(t), b_{\alpha^{\prime} \beta^{\prime}}^{+}\left(t^{\prime}\right)\right]=0} \\
{\left[b_{\alpha \beta}(t), b_{\alpha^{\prime} \beta^{\prime}}^{+}\left(t^{\prime}\right)\right]=\delta_{\varepsilon_{\alpha \beta}, \varepsilon_{\alpha^{\prime} \beta^{\prime}}} \delta\left(t-t^{\prime}\right) G^{\alpha \beta \alpha^{\prime} \beta^{\prime}}}
\end{gathered}
$$


where the constants $G^{\alpha \beta \alpha^{\prime} \beta^{\prime}}$ are given by

$$
G^{\alpha \beta \alpha^{\prime} \beta^{\prime}}=\int_{-\infty}^{\infty} d \tau \int d k g_{\alpha \beta}(\underline{k}) \overline{g_{\alpha^{\prime} \beta^{\prime}}(\underline{k})} e^{i \tau\left(\omega(\underline{k})-\epsilon_{\alpha \beta}\right)}=2 \pi \int d k g_{\alpha \beta}(k) g_{\alpha \beta}(k) \delta\left(\omega(\underline{k})-\varepsilon_{\alpha \beta}\right)
$$

The vacuum of the master fields $b_{\alpha \beta}(t)$ will be denoted by $\eta_{0}$ :

$$
b_{\alpha \beta}(t) \eta_{0}=0 \quad \forall \alpha \beta, \forall t
$$

The limit Hamiltonian is, then, see [3],

$$
H_{e b}^{(s l)}(t)=e^{2} \sum_{\alpha \beta}\left(a_{\alpha}^{+} a_{\beta} b_{\alpha \beta}(t)+\text { h.c. }\right)
$$

In this sense we say that $H_{e b}^{(s l)}(t)$ is the "stochastic limit" of $H_{e b}(t)$ in (2.16). Moreover, the wave operator in the stochastic limit satisfies the equation

$$
\partial_{t} U_{t}=-i H_{e b}^{(s l)}(t) U_{t}
$$

or, in integral form,

$$
U_{t}=\mathbb{1}-i \int_{0}^{t} H_{e b}^{(s l)}\left(t^{\prime}\right) U_{t^{\prime}} d t^{\prime}
$$

Finally, the stochastic limit of the (Heisenberg) time evolution of any observable $X$ of the system is:

$$
j_{t}(\tilde{X})=U_{t}^{+}\left(X \otimes \mathbb{1}_{R}\right) U_{t}
$$

where $\mathbb{1}_{R}$ is the identity of the reservoir. Since the $b_{\alpha \beta}(t)$ are quantum white noises, equation (2.29), and the corresponding differential equation for $j_{t}(\tilde{X})$, are singular equations and to give them a meaning we bring them in normal form. This normally ordered evolution equation is called the quantum Langevin equation. Its explicit form is:

$$
\begin{gathered}
\partial_{t} j_{t}(X)=e^{2} \sum_{\alpha \beta}\left\{j_{t}\left(\left[a_{\alpha}^{+} a_{\beta}, X\right] \Gamma_{-}^{\alpha \beta}-\Gamma_{-}^{\alpha \beta}\left[a_{\beta}^{+} a_{\alpha}, X\right]\right)\right\}+ \\
+i e^{2} \sum_{\alpha \beta}\left\{b_{\alpha \beta}^{+}(t) j_{t}\left(\left[a_{\beta}^{+} a_{\alpha}, X\right]\right)+j_{t}\left(\left[a_{\alpha}^{+} a_{\beta}, X\right]\right) b_{\alpha \beta}(t)\right\}
\end{gathered}
$$

where

$$
\begin{gathered}
\Gamma_{-}^{\alpha \beta}:=\sum_{\alpha^{\prime} \beta^{\prime}} \delta_{\varepsilon_{\alpha \beta}, \varepsilon_{\alpha^{\prime} \beta^{\prime}}} a_{\beta^{\prime}}^{+} a_{\alpha^{\prime}} G_{-}^{\alpha \beta \alpha^{\prime} \beta^{\prime}} \\
G_{-}^{\alpha \beta \alpha^{\prime} \beta^{\prime}}=\int_{-\infty}^{0} d \tau \int d k g_{\alpha \beta}(\underline{k}) \overline{g_{\alpha^{\prime} \beta^{\prime}}(\underline{k})} e^{i \tau\left(\omega(\underline{k})-\epsilon_{\alpha \beta}\right)}=
\end{gathered}
$$




$$
=\frac{1}{2} G^{\alpha \beta \alpha^{\prime} \beta^{\prime}}-i \text { P.P. } \int g_{\alpha \beta}^{(k)} \overline{g_{\alpha^{\prime} \beta^{\prime}}^{(k)}} \frac{1}{\omega_{k}-\varepsilon_{\alpha \beta}}
$$

The master equation is obtained by taking the mean value of (2.32) in the state $\eta_{0}^{(\xi)}=\eta_{0} \otimes \xi$, $\xi$ being a generic vector of the system. This gives

$$
\left\langle\partial_{t} j_{t}(X)\right\rangle_{\eta_{0}^{(\xi)}}=e^{2} \sum_{\alpha \beta}\left\langle j_{t}\left(\left[a_{\alpha}^{+} a_{\beta}, X\right] \Gamma_{-}^{\alpha \beta}-\Gamma_{-}^{\alpha \beta+}\left[a_{\beta}^{+} a_{\alpha}, X\right]\right)\right\rangle_{\eta_{0}^{(\xi)}}
$$

and from this we find for the generator

$$
L(X)=e \sum_{\alpha \beta \alpha^{\prime} \beta^{\prime}} \delta_{\varepsilon_{\alpha \beta}, \varepsilon_{\alpha^{\prime} \beta^{\prime}}}\left\{\left[a_{\alpha}^{+} a_{\beta}, X\right] a_{\beta^{\prime}}^{+} a_{\alpha^{\prime}} G_{-}^{\alpha \beta \alpha^{\prime} \beta^{\prime}}-a_{\alpha^{\prime}}^{+} a_{\beta^{\prime}}\left[a_{\beta}^{+} a_{\alpha}, X\right] \overline{G_{-}^{\alpha \beta \alpha^{\prime} \beta^{\prime}}}\right\}
$$

This expressions for $L(X)$ will be the starting point for our successive analysis.

\section{II.2 The current operator in second quantization}

The current is proportional to the sum of the velocities of the electrons:

$$
\vec{J}_{\Lambda}(t)=\alpha_{c} \sum_{i=1}^{N} \frac{d}{d t} \vec{R}_{i}(t)
$$

Here $\Lambda$ is the two-dimensional region corresponding to the physical layer, $\alpha_{c}$ is a proportionality constant which takes into account the electron charge, the area of the surface of the physical device and other physical quantities, and $\vec{R}_{i}(t)$ is the position operator for the $i$-th electron. Moreover $N$ is the number of electrons contained in $\Lambda$. Defining

$$
\vec{X}_{\Lambda}(t)=\sum_{i=1}^{N} \vec{R}_{i}(t)
$$

we simply write

$$
\vec{J}_{\Lambda}(t)=\alpha_{c} \dot{\vec{X}}_{\Lambda}(t)
$$

Since $\vec{X}_{\Lambda}(t)$ is a sum of single-electron operators its expression in second quantization is given by

$$
\vec{X}_{\Lambda}=\sum_{\gamma \mu} \vec{X}_{\gamma \mu} a_{\gamma}^{+} a_{\mu}
$$

where

$$
\vec{X}_{\gamma \mu}=\left\langle\psi_{\gamma}, \vec{X}_{\Lambda} \psi_{\mu}\right\rangle=\int \psi_{\gamma}(\underline{r}) \underline{r} \psi_{\mu}(\underline{r}) d r
$$

Recall that the $\psi_{\gamma}(\underline{r})$ are the single electron wave functions given in (2.14) and that $a_{\alpha}$ and $a_{\alpha}^{+}$ satisfy the anticommutation relations (2.10). In the LLL, $n_{\gamma}=n_{\mu}=0$, we find that, [3], 


$$
\begin{gathered}
X_{\gamma \mu}^{(1)}=\left(1-\delta_{p_{\mu} p_{\gamma}}\right)(-1)^{p_{\mu}-p_{\gamma}} L_{x} \frac{e^{-y_{p_{\mu} p_{\gamma}}^{2}}}{2 \pi i\left(p_{\mu}-p_{\gamma}\right)} \\
X_{\gamma \mu}^{(2)}=y_{0}^{\left(p_{\gamma}\right)} \delta_{p_{\mu} p_{\gamma}}
\end{gathered}
$$

where $y_{p_{\mu} p_{\gamma}}:=\sqrt{\frac{m \omega}{4 \hbar}}\left(y_{0}^{\left(p_{\mu}\right)}-y_{0}^{\left(p_{\gamma}\right)}\right)=\frac{\pi}{L_{x}} \sqrt{\frac{\hbar}{m \omega}}\left(p_{\mu}-p_{\gamma}\right)$.

To show how these results can be useful in the computation of the electron current we start noticing that, if $\varrho$ is a state of the electron system, then

$$
\left\langle\vec{J}_{\Lambda}(t)\right\rangle_{\varrho}=\alpha_{c}\left\langle\frac{d}{d t} \vec{X}_{\Lambda}(t)\right\rangle_{\varrho}=\alpha_{c}\left\langle L\left(\vec{X}_{\Lambda}(t)\right)\right\rangle_{\varrho}=\alpha_{c} \operatorname{Tr}\left(\varrho L\left(\vec{X}_{\Lambda}(t)\right)\right)
$$

The vector $\left\langle\vec{J}_{\Lambda}(t)\right\rangle_{\varrho}$ will be computed now for a particular class of states $\varrho$, and we will use this result to get the expressions for the conductivity and for the resistivity tensor.

To do this we begin computing the electric current. We first need to find $L\left(\vec{X}_{\Lambda}\right), L$ being the generator given in (2.36). Since $\vec{X}_{\Lambda}=\vec{X}_{\Lambda}^{\dagger}$, we have

$$
L\left(\vec{X}_{\Lambda}\right)=L_{1}\left(\vec{X}_{\Lambda}\right)+h . c .,
$$

where, as we find after a few computations,

$$
L_{1}\left(\vec{X}_{\Lambda}\right)=e^{2} \sum_{\alpha \beta \alpha^{\prime} \beta^{\prime}, \gamma} \delta_{\epsilon_{\alpha \beta}, \epsilon_{\alpha^{\prime} \beta^{\prime}}} G_{-}^{\alpha \beta \alpha^{\prime} \beta^{\prime}}\left(\vec{X}_{\beta \gamma} a_{\alpha}^{+} a_{\gamma} a_{\beta^{\prime}}^{+} a_{\alpha^{\prime}}-\vec{X}_{\gamma \alpha} a_{\gamma}^{+} a_{\beta} a_{\beta^{\prime}}^{+} a_{\alpha^{\prime}}\right)
$$

We consider here a situation of zero temperature and we compute the mean value of $L_{1}\left(\vec{X}_{\Lambda}\right)$ on a Fock $N$-particle state $\psi_{I}$ :

$$
\psi_{I}=a_{i_{1}}^{+} \ldots a_{i_{N_{I}}}^{+} \psi_{0}, \quad i_{k} \neq i_{l}, \forall k \neq l
$$

where $I$ is a set of possible quantum numbers, $N_{I}$ is the number of electrons in $I$ and $\psi_{0}$ is the vacuum vector of the fermionic operators, $a_{\alpha} \psi_{0}=0$ for all $\alpha$. The order of the elements of $I$ is important to fix uniquely the phase of $\psi_{I}$. Equation (2.44) gives now

$$
\left.\left\langle\psi_{I}, \vec{J}_{\Lambda}(t) \psi_{I}\right\rangle\right|_{t=0}=\alpha_{c}\left\langle\psi_{I}, L\left(\vec{X}_{\Lambda}\right) \psi_{I}\right\rangle
$$

Introducing now the characteristic function of the set $I$,

$$
\chi_{I}(\alpha)=\left\{\begin{array}{l}
1 \text { if } \alpha \in I \\
0 \text { if } \alpha \notin I,
\end{array}\right.
$$

we get

$$
\left\langle a_{\gamma}^{\dagger} a_{\alpha} \psi_{I}, a_{\beta^{\prime}}^{\dagger} a_{\alpha^{\prime}} \psi_{I}\right\rangle=\delta_{\alpha \gamma} \delta_{\alpha^{\prime} \beta^{\prime}} \chi_{I}(\alpha) \chi_{I}\left(\alpha^{\prime}\right)+\delta_{\alpha \alpha^{\prime}} \delta_{\gamma \beta^{\prime}} \chi_{I}(\alpha)\left(1-\chi_{I}(\gamma)\right),
$$


see [3] for the details. Using this equality, together with

$$
\delta_{\varepsilon_{\alpha \beta}, \varepsilon_{\alpha^{\prime} \alpha^{\prime}}}=\delta_{\varepsilon_{\alpha}, \varepsilon_{\beta}} \quad \delta_{\varepsilon_{\alpha \beta}, \varepsilon_{\alpha \beta^{\prime}}}=\delta_{\varepsilon_{\beta}, \varepsilon_{\beta^{\prime}}}
$$

we find that the average current is proportional to

$$
\left\langle L\left(\vec{X}_{\Lambda}\right)\right\rangle_{\psi_{I}}=\mathcal{L}_{1}\left(\vec{X}_{\Lambda}\right)+\mathcal{L}_{2}\left(\vec{X}_{\Lambda}\right)
$$

where we isolate two contributions of different structure:

$$
\begin{aligned}
\mathcal{L}_{1}\left(\vec{X}_{\Lambda}\right)= & e^{2} \sum_{\alpha \beta \alpha^{\prime}} \delta_{\varepsilon_{\alpha}, \varepsilon_{\beta}}\left\{\chi_{I}(\alpha)-\chi_{I}(\beta)\right\} \chi_{I}\left(\alpha^{\prime}\right)\left(\vec{X}_{\alpha \beta} \overline{G_{-}^{\alpha \beta \alpha^{\prime} \alpha^{\prime}}}+\vec{X}_{\beta \alpha} G_{-}^{\alpha \beta \alpha^{\prime} \alpha^{\prime}}\right) \\
\mathcal{L}_{2}\left(\vec{X}_{\Lambda}\right)= & e^{2} \sum_{\alpha \beta \beta^{\prime}} \delta_{\varepsilon_{\beta}, \varepsilon_{\beta^{\prime}}}\left\{\vec{X}_{\beta \beta^{\prime}}\left[G_{-}^{\alpha \beta \alpha \beta^{\prime}} \chi_{I}(\alpha)\left(1-\chi_{I}\left(\beta^{\prime}\right)\right)-\overline{G_{-}^{\beta \alpha \beta^{\prime} \alpha}} \chi_{I}\left(\beta^{\prime}\right)\left(1-\chi_{I}(\alpha)\right)\right]-\right. \\
& \left.-\vec{X}_{\beta^{\prime} \beta}\left[G_{-}^{\beta \alpha \beta^{\prime} \alpha} \chi_{I}\left(\beta^{\prime}\right)\left(1-\chi_{I}(\alpha)\right)-\overline{G_{-}^{\alpha \beta \alpha \beta^{\prime}}} \chi_{I}(\alpha)\left(1-\chi_{I}\left(\beta^{\prime}\right)\right)\right]\right\}
\end{aligned}
$$

Using equations $(2.42),(2.43)$ for $X_{\gamma \mu}^{(i)}$ we are able to obtain $\mathcal{L}_{1}\left(X_{\Lambda}^{(i)}\right)$ and $\mathcal{L}_{2}\left(X_{\Lambda}^{(i)}\right)$ for $i=1,2$. First of all we can show that, even if $\mathcal{L}_{1}\left(X_{\Lambda}^{(1)}\right)$ is not zero, nevertheless it does not depend on the electric field. Therefore

$$
\frac{\partial}{\partial E} \mathcal{L}_{1}\left(X_{\Lambda}^{(1)}\right)=0
$$

Secondly, the computation of $\mathcal{L}_{2}\left(X_{\Lambda}^{(1)}\right)$ gives rise to an interesting phenomenon: due to the definition of $X_{\gamma \mu}^{(1)}$, the sum in (2.53) is different from zero only if $p_{\beta} \neq p_{\beta^{\prime}}$. Moreover, we also must have $\varepsilon_{\beta}=\varepsilon_{\beta^{\prime}}$, that is

$$
n_{\beta}-n_{\beta^{\prime}}=\frac{2 \pi e E}{m \omega^{2} L_{x}}\left(p_{\beta^{\prime}}-p_{\beta}\right)
$$

This equality can be satisfied in two different ways: let us denote $\mathcal{R}$ the set of all possible quotients of the form $\left(n_{\beta}-n_{\beta^{\prime}}\right) /\left(p_{\beta^{\prime}}-p_{\beta}\right)$. This set, in principle, coincides with the set of the rational numbers. Therefore $0 \in \mathcal{R}$. Then

1) if $\frac{2 \pi e E}{m \omega^{2} L_{x}}$ is not in $\mathcal{R},(2.55)$ can be satisfied only if $\beta=\beta^{\prime}$. But this condition implies in particular that $p_{\beta}=p_{\beta^{\prime}}$, and we know already that whenever this condition holds, then $X_{\beta \beta^{\prime}}^{(1)}=0$, so that $\mathcal{L}_{2}\left(X_{\Lambda}^{(1)}\right)=0$. 
2) If $\frac{2 \pi e E}{m \omega^{2} L_{x}}$ is in $\mathcal{R}$, then we have two possibilities: the first one is again

$$
\beta=\beta^{\prime}
$$

which, as we have just shown, does not contribute to $\mathcal{L}_{2}\left(X_{\Lambda}^{(1)}\right)$. The second is

$$
\frac{n_{\beta}-n_{\beta^{\prime}}}{p_{\beta^{\prime}}-p_{\beta}}=\frac{2 \pi e E}{m \omega^{2} L_{x}}
$$

which gives a non trivial contribution to the current.

Therefore, we can state the following

Proposition. In the context of our model there exists a set of rational numbers $\mathcal{R}$ with the following property: if the electric and the magnetic fields are such that the quotient

$$
\frac{2 \pi e E}{m \omega^{2} L_{x}}
$$

does not belong to $\mathcal{R}$ then

$$
\left\langle J_{\Lambda}^{(1)}(t)\right\rangle_{\psi_{I}}=0
$$

On the other hand, if condition (2.56) is satisfied, we can conclude that the $\operatorname{sum} \sum_{\alpha \beta \beta^{\prime}} \delta_{\varepsilon_{\beta}, \varepsilon_{\beta^{\prime}}}(\ldots)$ in $(2.53)$ can be replaced by

$$
\sum_{\alpha \beta \beta^{\prime}} \delta_{\varepsilon_{\beta}, \varepsilon_{\beta^{\prime}}}(\ldots)=\sum_{\alpha} \sum_{\beta \beta^{\prime}}^{\prime}(\ldots)
$$

where $\sum_{\alpha} \sum_{\beta \beta}^{\prime}$ means that the sum is extended to all the $\alpha$ and to those $\beta$ and $\beta^{\prime}$ with $p_{\beta} \neq p_{\beta^{\prime}}$ satisfying (2.56) (which automatically implies that $\varepsilon_{\beta}=\varepsilon_{\beta^{\prime}}$ ).

Since, as it is easily seen, $g_{\alpha \beta}(k) \overline{g_{\alpha^{\prime} \beta^{\prime}}(\underline{k})}$ does not depend on $\vec{E}$, we find that

$$
\frac{\partial}{\partial E} G_{-}^{\alpha \beta \alpha^{\prime} \beta^{\prime}}=-i \frac{h e}{m \omega L_{x}}\left(p_{\alpha}-p_{\beta}\right) \Lambda_{-}^{\alpha \beta \alpha^{\prime} \beta^{\prime}}
$$

where

$$
\Lambda_{-}^{\alpha \beta \alpha^{\prime} \beta^{\prime}}=\int_{-\infty}^{0} d \tau \int d k g_{\alpha \beta}(\underline{k}) \overline{g_{\alpha^{\prime} \beta^{\prime}}(\underline{k})} e^{i \tau\left(\omega(\underline{k})-\varepsilon_{\alpha \beta}\right)}
$$

so that, using also (2.57), we get

$$
\frac{\partial}{\partial E} \mathcal{L}_{2}\left(X_{\Lambda}^{(1)}\right)=\frac{h e}{m \omega L_{x}} \Theta_{x}
$$


where

$$
\begin{gathered}
\Theta_{x}:=\sum_{\alpha} \sum_{\beta \beta^{\prime}}^{\prime}\left(p_{\beta}-p_{\alpha}\right) \tilde{x}_{\beta \beta^{\prime}}^{(1)}\left\{\chi_{I}(\alpha)\left(1-\chi_{I}\left(\beta^{\prime}\right)\right) \cdot\left(\Lambda_{-}^{\alpha \beta \alpha \beta^{\prime}}+\overline{\Lambda_{-}^{\alpha \beta \alpha \beta^{\prime}}}\right)\right. \\
\left.-\chi_{I}\left(\beta^{\prime}\right)\left(1-\chi_{I}(\alpha)\right)\left(\Lambda_{-}^{\beta \alpha \beta^{\prime} \alpha}+\overline{\Lambda_{-}^{\beta \alpha \beta^{\prime} \alpha}}\right)\right\}
\end{gathered}
$$

and

$$
\tilde{x}_{\beta \beta^{\prime}}^{(1)}=i X_{\beta \beta^{\prime}}^{(1)} \quad(\in \mathbf{R})
$$

Therefore we conclude that

$$
\frac{\partial}{\partial E}\left\langle J_{\Lambda}^{(1)}(t)\right\rangle_{\psi_{I}}=\frac{\alpha_{c} h e^{3}}{m \omega L_{x}} \Theta_{x}
$$

Let us now compute the second component of the average current: $\left\langle\psi_{I}, L\left(X_{\Lambda}^{(2)}\right) \psi_{0}\right\rangle=$ $\mathcal{L}_{1}\left(X_{\Lambda}^{(2)}\right)+\mathcal{L}_{2}\left(X_{\Lambda}^{(2)}\right)$.

The first contribution is easily shown, from (2.52) and (2.43), to be identically zero, since

$$
\delta_{\varepsilon_{\alpha}, \varepsilon_{\beta}} \delta_{p_{\alpha} p_{\beta}}=\delta_{\alpha \beta}
$$

On the contrary the second term, $\mathcal{L}_{2}\left(X_{\Lambda}^{(2)}\right)$, is different from zero and it has an interesting expression: in fact, due to the factor $\delta_{p_{\mu}, p_{\gamma}}$, the only non trivial contributions in the sum $\sum_{\beta \beta^{\prime}} \delta_{\varepsilon_{\beta}, \varepsilon_{\beta^{\prime}}}$, in $(2.53)$, are exactly those with $\beta=\beta^{\prime}$. Taking all this into account, we find that

$$
\mathcal{L}_{2}\left(X_{\Lambda}^{(2)}\right)=e^{2} \sum_{\alpha \beta}\left(y_{0}^{\left(p_{\beta}\right)}-y_{0}^{\left(p_{\alpha}\right)}\right) \chi_{I}(\alpha)\left(1-\chi_{I}(\beta)\right)\left(G_{-}^{\alpha \beta \alpha \beta}+\overline{G_{-}^{\alpha \beta \alpha \beta}}\right)
$$

which is different from zero. Furthermore, using (2.58), we get

$$
\frac{\partial}{\partial E} \mathcal{L}_{2}\left(X_{\Lambda}^{(2)}\right)=-2 e^{3}\left(\frac{h}{m \omega L_{x}}\right)^{2} \Theta_{y}
$$

were we have defined

$$
\Theta_{y}=\sum_{\alpha, \beta}\left(p_{\alpha}-p_{\beta}\right)^{2} \chi_{I}(\alpha)\left(1-\chi_{I}(\beta)\right) \operatorname{Im}\left(\Lambda_{-}^{\alpha \beta \alpha \beta}\right)
$$

and $\Lambda_{-}^{\alpha \beta \alpha \beta}$ is given by (2.59). If we call now

$$
\begin{gathered}
j_{x, E}=\left.\frac{\partial\left\langle J_{\Lambda}^{(1)}(t)\right\rangle_{\psi_{I}}}{\partial E}\right|_{t=0}=\alpha_{c} \frac{\partial\left\langle L\left(X_{\Lambda}^{(1)}\right)\right\rangle_{\psi_{I}}}{\partial E} \\
j_{y, E}=\left.\frac{\partial\left\langle J_{\Lambda}^{(2)}(t)\right\rangle_{\psi_{I}}}{\partial E}\right|_{t=0}=\alpha_{c} \frac{\partial\left\langle L\left(X_{\Lambda}^{(2)}\right)\right\rangle_{\psi_{I}}}{\partial E},
\end{gathered}
$$


we obtain the conductivity tensor (see [9])

$$
\sigma_{x x}=\sigma_{y y}=j_{y, E}, \quad \sigma_{x y}=-\sigma_{y x}=j_{x, E}
$$

and the resistivity tensor

$$
\rho_{x x}=\rho_{y y}=\frac{\sigma_{y y}}{\sigma_{y y}^{2}+\sigma_{x y}^{2}}, \quad \rho_{x y}=-\rho_{y x}=\frac{\sigma_{x y}}{\sigma_{y y}^{2}+\sigma_{x y}^{2}}
$$

After minor computations we conclude that

$$
\begin{gathered}
\rho_{x y}=\left\{\begin{array}{lc}
0 & \text { if } \frac{2 \pi e E}{m \omega^{2} L_{x}} \notin \mathcal{R} \\
\frac{m \omega L_{x}}{2 e^{3} h \alpha_{c}} \frac{\Theta_{x}}{\left[\Theta_{x}^{2}+\left(\frac{h}{m \omega L_{x}}\right)^{2} \Theta_{y}^{2}\right]} & \text { if } \frac{2 \pi e E}{m \omega^{2} L_{x}} \in \mathcal{R},
\end{array}\right. \\
\rho_{x x}=\left\{\begin{array}{lr}
-\left(\frac{m \omega L_{x}}{h}\right)^{2} \frac{1}{2 \alpha_{c} e^{3} \Theta_{y}} & \text { if } \frac{2 \pi e E}{m \omega^{2} L_{x}} \notin \mathcal{R} \\
-\frac{1}{2 e^{3} \alpha_{c}} \frac{\Theta_{y}}{\left[\Theta_{x}^{2}+\left(\frac{h}{m \omega L_{x}}\right)^{2} \Theta_{y}^{2}\right]} & \text { if } \frac{2 \pi e E}{m \omega^{2} L_{x}} \in \mathcal{R},
\end{array}\right.
\end{gathered}
$$

Let us now comment these results which are consequences of the basic relation (2.56). As it is evident from the formula above, the fact that the fine tuning condition (FTC) $\left(\frac{2 \pi e E}{m \omega^{2} L_{x}} \in \mathcal{R}\right)$ is satisfied implies that $\rho_{x y} \neq 0$, so that the resistivity tensor is non-diagonal. Vice-versa, if the FTC is not satisfied, then $\rho=\rho_{x x} \mathbb{1}, \mathbb{1}$ being the $2 \times 2$ identity matrix. This implies that, whenever the FTC holds, then the $x$-component of the mean value of the density current operator is in general different from zero, while it is necessarily zero if the FTC is not satisfied.

If the physical system is prepared in such a way that $\frac{2 \pi e E}{m \omega^{2} L_{x}} \in \mathcal{R}$, then an experimental device should be able to measure a non zero current along the $x$-axis. Otherwise, this current should be zero whenever $\frac{2 \pi e E}{m \omega^{2} L_{x}} \notin \mathcal{R}$. A crucial point is now the determination of the set $\mathcal{R}$, of rational numbers. From a mathematical point of view, all the natural integers $n_{\alpha}$ and all the relative integer $p_{\alpha}$ are allowed. However physics restricts the experimentally relevant values to a finite set. In fact eigenstates corresponding to high values of $n_{\alpha}$ and $p_{\alpha}$ are energetically not favoured because the associated eigenenergies $\varepsilon_{n_{\alpha} p_{\alpha}}$ in (2.14) increases and the probabilities of finding an electron in the corresponding eigenstate decrease (this is a generalization of the standard argument which restrict the analysis of the fractional QHE to the first few Landau levels). Moreover, high positive values of $-p_{\alpha}$ are not compatible with the fact that $H_{0}$ must be bounded from below, to be a honest Hamiltonian.

Therefore, in formula (2.56) not all the rational numbers are physically allowed but only those compatible with the above constraints. For this reason it is quite reasonable to expect that the set $\mathcal{R}$ consists only of a finite set of rational values. Of course, the determination of this set strongly depends on the physics of the experimental setting. 
Finally, let us remark that the sharp values of the magnetic field involved in the FTC may be a consequence of the approximation intrinsic in the stochastic limit procedure, which consists in taking $\lambda \rightarrow 0$ and $t \rightarrow \infty$. In intermediate regions $(\lambda>0$ and $t<\infty)$, it is not hard to imagine that the $\delta$-function giving rise to the FTC becomes a smoother function, and that real plateaux, closer to what is observed in the QHE, appear.

Also, under special assumptions on the $B$-dependence of $\Theta_{x}$ and $\Theta_{y}$, together with some reasonable physical constraint on the value of the magnetic field, it is not difficult to check that $\rho_{x x}$ has plateaux corresponding to the zeros of $\rho_{x y}$ and that, outside of these plateaux, it grows linearly with $B$.

\section{Laser Models}

In two recent papers, [12, 13], a dissipative laser model has been introduced and analyzed in some details. In particular in [12] (AS in the following) the rigorous definition of the unbounded generator of the model, which consists of a sum of a free radiation and a free matter generator plus a matter-radiation term, is given and the existence of the thermodynamical limit of the dynamics of some macroscopic observables is deduced. Moreover, the analysis of this dynamics shows that two phase transitions occur in the model, depending on the value of a certain pumping strength. In [13] the analysis has been continued paying particular attention to the existence of the dynamics of the microscopic observables, which are only those of the matter since, in the thermodynamical limit, we proved that the field of the radiation becomes classical. Also, the existence of a transient has been proved and an entropy principle has been deduced.

On the other hand, in a series of papers $[14,15]$ culminating with the fundamental work by Hepp and Lieb [16] (HL in the following) many conservative models of matter interacting with radiation were proposed. In particular, in [16] the authors have introduced a model of an open system of matter and of a single mode of radiation interacting among themselves and with their own (bosonic) reservoirs, but, to simplify the treatment, they have considered a simplified version in which the matter bosonic reservoir is replaced by a fermionic one. This is to avoid dealing with unbounded operators. This is what they call the Dicke-Haken-Lax model (DHL model in the following).

In $[12,13]$ the relation between the AS model and a many mode version of the HL model is claimed: of course, since no reservoir appear in the semigroup formulation as given by [12], this claim is reasonable but it is not clear the explicit way in which HL should be related to AS. Here we will show that the relation between the two models is provided by (a slightly 
modified version of) the stochastic limit (SL). In particular, if we start with the physical AS system (radiation and matter) and we introduce in a natural way two reservoirs (one is not enough!) for the matter and another reservoir for the radiation, then the SL of the hamiltonian for the new system constructed in this way returns back the original AS generator, under very reasonable hypotheses. Moreover, the model which we have constructed ad hoc to get this generator surprisingly coincides with the HL laser model, [16]. This is the content of Subsection III.1, while in Subsection III.2 we will consider the SL of the DHL model, [16, 17]. We will find that, even if the form of the generator apparently differs from the one by AS, under certain conditions on the coefficients which define the model, the equations of motion for the observables of the matter-radiation system coincide with the ones given in AS.

Let us discuss the main characteristics of the three physical models which will be considered in this section. In particular, we will only give the definition of the hamiltonians for the HL and the DHL models and the expression of the generator for the AS model, without even mentioning mathematical details like, for instance, those related to the domain problem intrinsic with all these models due to the presence of bosonic operators. We refer to the original papers for these and further details which are not relevant in this work.

We begin with the AS model.

This model is a dissipative quantum system, $\Sigma^{(N)}$, consisting of a chain of $2 N+1$ identical two-level atoms interacting with an $n$-mode radiation field, $n$ fixed and finite. We build the model from its constituent parts starting with the single atom.

This is assumed to be a two-state atom or spin, $\Sigma_{a t}$. Its algebra of observables, $\mathcal{A}_{a t}$, is that of the two-by-two matrices, spanned by the Pauli matrices $\left(\sigma_{x}, \sigma_{y}, \sigma_{z}\right)$ and the identity, $\mathbb{1}$. They satisfy the relations

$$
\sigma_{x}^{2}=\sigma_{y}^{2}=\sigma_{z}^{2}=\mathbb{1} ; \sigma_{x} \sigma_{y}=i \sigma_{z} \text {, etc. }
$$

We define the spin raising and lowering operators

$$
\sigma_{ \pm}=\frac{1}{2}\left(\sigma_{x} \pm i \sigma_{y}\right)
$$

We assume that the atom is coupled to a pump and a sink, and that its dynamics is given by a one-parameter semigroup $\left\{T_{a t}(t) \mid t \in \mathbf{R}_{+}\right\}$of completely positive, identity preserving contractions of $\mathcal{A}_{a t}$, whose generator, $L_{a t}$, is of the following form.

$$
L_{a t} \sigma_{ \pm}=-\left(\gamma_{1} \mp i \epsilon\right) \sigma_{ \pm} ; L_{a t} \sigma_{z}=-\gamma_{2}\left(\sigma_{z}-\eta I\right),
$$

where $\epsilon(>0)$ is the energy difference between the ground and excited states of the atom, and the $\gamma$ 's and $\eta$ are constants whose values are determined by the atomic coupling to the energy 
source and sink, and are subject to the restrictions that

$$
0<\gamma_{2} \leq 2 \gamma_{1} ;-1 \leq \eta \leq 1
$$

The matter consists of $2 N+1$ non-interacting copies of $\Sigma_{a t}$, located at the sites $r=-N, \ldots, N$ of the one-dimensional lattice $\mathbf{Z}$. Thus, at each site, $r$, there is a copy, $\Sigma_{r}$, of $\Sigma_{a t}$, whose algebra of observables, $\mathcal{A}_{r}$, and dynamical semigroup, $T_{r}$, are isomorphic with $\mathcal{A}_{a t}$ and $T_{a t}$, respectively. We denote by $\sigma_{r, u}$ the copy of $\sigma_{u}$ at $r$, for $u=x, y, z, \pm$.

We define the algebra of observables, $\mathcal{A}^{(N)}$, and the dynamical semigroup, $T_{\text {mat }}^{(N)}$, of the matter to be $\otimes_{r=-N}^{N} \mathcal{A}_{r}$ and $\otimes_{r=-N}^{N} T_{r}$, respectively. Thus, $\mathcal{A}^{(N)}$ is the algebra of linear transformations of $\mathrm{C}^{4 N+2}$. We identify elements $A_{r}$ of $\mathcal{A}_{r}$ with those of $\mathcal{A}^{(N)}$ given by their tensor products with the identity operators attached to the remaining sites. Under this identification, the commutant, $\mathcal{A}_{r}^{\prime}$, of $\mathcal{A}_{r}$ is the tensor product $\otimes_{s \neq r} \mathcal{A}_{s}$. The same identification will be implicitly assumed for the other models.

It follows from these specifications that the generator, $L_{\mathrm{mat}}^{(N)}$, of $T_{\text {mat }}^{(N)}$ is given by the formula

$$
L_{\text {mat }}^{(N)}=\sum_{l \in I_{N}} L_{l}
$$

where $I_{N}=\{-N, \ldots,-1,0,1, . ., N\}$. Here

$$
\begin{gathered}
L_{r} \sigma_{r, \pm}=-\left(\gamma_{1} \mp i \epsilon\right) \sigma_{r, \pm} ; \quad L_{r} \sigma_{r, z}=-\gamma_{2}\left(\sigma_{r, z}-\eta \mathbb{1}\right) \\
\text { and } L_{r}\left(A_{r} A_{r}^{\prime}\right)=\left(L_{r} A_{r}\right) A_{r}^{\prime} \forall A_{r} \in \mathcal{A}_{r}, A_{r}^{\prime} \in \mathcal{A}_{r}^{\prime}
\end{gathered}
$$

We assume, furthermore, that the radiation field consists of $n(<\infty)$ modes, represented by creation and destruction operators $\left\{a_{l}^{\star}, a_{l} \mid l=0, \ldots, n-1\right\}$ in a Fock-Hilbert space $\mathcal{H}_{\text {rad }}$ as defined by the standard specifications that (a) these operators satisfy the CCR,

$$
\left[a_{l}, a_{m}^{\star}\right]=\delta_{l m} \mathbb{1} ;\left[a_{l}, a_{m}\right]=0,
$$

and (b) $\mathcal{H}_{\text {rad }}$ contains a (vacuum) vector $\Phi$, that is annihilated by each of the $a$ 's and is cyclic w.r.t. the algebra of polynomials in the $a^{\star}$ 's.

The formal generator of the semigroup $T_{\text {rad }}$ of the radiation is

$$
L_{\mathrm{rad}}=\sum_{l=0}^{n-1}\left(i \omega_{l}\left[a_{l}^{\star} a_{l}, .\right]+2 \kappa_{l} a_{l}^{\star}(.) a_{l}-\kappa_{l}\left\{a_{l}^{\star} a_{l}, .\right\}\right),
$$

where $\{.,$.$\} denotes anticommutator, and the frequencies, \omega_{l}$, and the damping constants, $\kappa_{l}$, are positive. We refer to [12] for a rigorous definition of $L_{\mathrm{rad}}$. 
The composite (finite) system is simply the coupled system, $\Sigma^{(N)}$, comprising the matter and the radiation. We assume that its algebra of observables, $\mathcal{B}^{(N)}$, is the tensor product $\mathcal{A}^{(N)} \otimes \mathcal{R}$, where $\mathcal{R}$ is the ${ }^{\star}$-algebra of polynomials in the $a^{\prime}$ 's, $a^{\star}$ 's and the Weyl operators. Thus, $\mathcal{B}^{(N)}$, like $\mathcal{R}$, is an algebra of both bounded and unbounded operators in the Hilbert space $\mathcal{H}^{(N)}:=\mathbf{C}^{4 N+2} \otimes \mathcal{H}_{\text {rad }}$. We shall identify elements $A, R$, of $\mathcal{A}^{(N)}, \mathcal{R}$, with $A \otimes \mathbb{1}_{\text {rad }}$ and $\mathbb{1}_{\text {mat }} \otimes R$, respectively, with obvious notation.

We assume that the matter-radiation coupling is dipolar and is given by the interaction Hamiltonian

$$
H_{\mathrm{int}}^{(N)}=\sum_{r \in I_{N}}\left(\sigma_{r,+} \phi_{r}^{(N)}+\text { h.c. }\right),
$$

where we have introduced the so-called radiation field, $\phi^{(N)}$, whose value at the site $r$ is

$$
\phi_{r}^{(N)}=-i(2 N+1)^{-1 / 2} \sum_{l=0}^{n-1} \lambda_{l} a_{l} \exp (2 \pi i l r / n) .
$$

Here the $\lambda$ 's are real-valued, $N$-independent coupling constants.

Among the other results contained in [12], one of the most relevant is that the map

$$
L^{(N)}=L_{\mathrm{mat}}^{(N)}+L_{\mathrm{rad}}+i\left[H_{\mathrm{int}}^{(N)}, .\right]
$$

is really the generator of a $N$-depending semigroup, $T^{(N)}$, regardless of the unbounded nature of both $L_{\text {rad }}$ and $H_{\text {int }}^{(N)}$.

Let us now introduce the $n$-modes version of the HL model, [16]. The HL hamiltonian for the $2 N+1$ atoms and for the $n$ modes of the radiation can be written as follows:

$$
H=H^{(S)}+H^{(R)},
$$

where "S" refers to the system (radiation+matter) and "R" to the reservoir. The hamiltonian of the system is

$$
\begin{aligned}
& H^{(S)}=\omega_{R} \sum_{j=0}^{n-1} a_{j}^{\dagger} a_{j}+\mu \sum_{l \in I_{N}} \sigma_{l, z}+\frac{\alpha}{\sqrt{2 N+1}} \sum_{j=0}^{n-1} \sum_{l \in I_{N}}\left(\sigma_{l,+} a_{j} e^{2 \pi i j l / n}+\sigma_{l,-} a_{j}^{\dagger} e^{-2 \pi i j l / n}\right)+ \\
& +\frac{\beta}{\sqrt{2 N+1}} \sum_{j=0}^{n-1} \sum_{l \in I_{N}}\left(\sigma_{l,+} a_{j}^{\dagger} e^{-2 \pi i j l / n}+\sigma_{l,-} a_{j} e^{2 \pi i j l / n}\right)
\end{aligned}
$$

[4]. Notice that the presence of $\beta$ means that we are not restricting our model to the rotating wave approximation, (RWA). 
The hamiltonian for the reservoir contains two main contributions, one related to the two reservoirs of the matter and one to the reservoir of the radiation. We have:

$$
H^{(R)}=H^{(P)}+\sum_{l \in I_{N}} H_{l}^{(A)}
$$

where

$$
H^{(P)}=\sum_{j=0}^{n-1} \int d k \omega_{r, j}(k) r_{j}(k)^{\dagger} r_{j}(k)+\sqrt{\alpha} \sum_{j=0}^{n-1}\left(r_{j}^{\dagger}\left(\bar{g}_{j}\right) a_{j}+r_{j}\left(g_{j}\right) a_{j}^{\dagger}\right)
$$

and

$$
H_{l}^{(A)}=\sum_{s=1}^{2} \int d k \omega_{m_{s}}(k) m_{s, l}^{\dagger}(k) m_{s, l}(k)+\sqrt{\alpha}\left(m_{1, l}^{\dagger}\left(\bar{h}_{1}\right) \sigma_{l,-}+h . c .\right)+\sqrt{\alpha}\left(m_{2, l}^{\dagger}\left(\bar{h}_{2}\right) \sigma_{l,+}+h . c .\right)
$$

We notice that:

1) we are using the notation: $r_{j}\left(g_{j}\right)=\int d k r_{j}(k) g_{j}(k)$ and $r_{j}^{\dagger}\left(\bar{g}_{j}\right)=\int d k r_{j}^{\dagger}(k) \bar{g}_{j}(k)$. Here $d k$ is a shortcut notation for $d \underline{k}^{3}$.

2) the functions $g_{j}$ and $h_{1,2}$ are introduced by HL to regularize the bosonic fields $r_{j}(k)$ and $m_{(1,2), l}(k)$.

3 ) in this model two independent reservoirs, $m_{1, l}(k)$ and $m_{2, l}(k)$, are introduced for (each atom of) the matter, while only one, $r_{j}(k)$, is used for (each mode of) the radiation. This result will be recovered also in our approach.

4) the coupling constant $\sqrt{\alpha}$ is written explicitly for later convenience.

The role of each term of the hamiltonian above is evident. We rewrite $H$ as a sum of a free and of an interaction part, in the following way:

$$
H=H_{0}+\sqrt{\alpha} H_{I}
$$

where

$$
H_{0}=\omega_{R} \sum_{j=0}^{n-1} a_{j}^{\dagger} a_{j}+\mu \sum_{l \in I_{N}} \sigma_{l, z}+\sum_{l \in I_{N}} \sum_{s=1}^{2} \int d k \omega_{m_{s}}(k) m_{s, l}^{\dagger}(k) m_{s, l}(k)+\sum_{j=0}^{n-1} \int d k \omega_{r, j}(k) r_{j}(k)^{\dagger} r_{j}(k)
$$

and

$$
H_{I}=\sum_{j=0}^{n-1}\left(r_{j}^{\dagger}\left(\bar{g}_{j}\right) a_{j}+r_{j}\left(g_{j}\right) a_{j}^{\dagger}\right)+\sum_{l \in I_{N}}\left[\left(m_{1, l}^{\dagger}\left(\bar{h}_{1}\right) \sigma_{l,-}+h . c .\right)+\left(m_{2, l}^{\dagger}\left(\bar{h}_{2}\right) \sigma_{l,+}+\text { h.c. }\right)\right]+
$$




$$
\begin{aligned}
& +\frac{\sqrt{\alpha}}{\sqrt{2 N+1}} \sum_{j=0}^{n-1} \sum_{l \in I_{N}}\left(\sigma_{l,+} a_{j} e^{2 \pi i j l / n}+\sigma_{l,-} a_{j}^{\dagger} e^{-2 \pi i j l / n}\right)+ \\
& +\frac{\beta}{\sqrt{\alpha(2 N+1)}} \sum_{j=0}^{n-1} \sum_{l \in I_{N}}\left(\sigma_{l,+} a_{j}^{\dagger} e^{-2 \pi i j l / n}+\sigma_{l,-} a_{j} e^{2 \pi i j l / n}\right) .
\end{aligned}
$$

The only non trivial commutation relations, which are different from the ones already given in $(3.1,3.7)$, are:

$$
\left[r_{j}(k), r_{l}\left(k^{\prime}\right)^{\dagger}\right]=\delta_{j, l} \delta\left(k-k^{\prime}\right), \quad\left[m_{s, l}(k), m_{s^{\prime}, l^{\prime}}^{\dagger}\left(k^{\prime}\right)\right]=\delta_{s, s^{\prime}} \delta_{l, l^{\prime}} \delta\left(k-k^{\prime}\right)
$$

Finally, let us introduce the DHL model. The main difference, [17], consists in the use of a fermionic reservoir for the matter, and for this reason the Pauli matrices of both AS and HL are replaced by fermionic operators as described in details, for instance, in [17], [4]. Again we have

$$
H=H_{0}+\lambda H_{I}
$$

where, this time,

$$
\begin{gathered}
H_{0}=\omega_{R} \sum_{j=0}^{n-1} a_{j}^{\dagger} a_{j}+\mu \sum_{l \in I_{N}}\left(b_{+, l}^{\dagger} b_{+, l}-b_{-, l}^{\dagger} b_{-, l}\right)+\sum_{j=0}^{n-1} \int d k \omega_{r, j}(k) r_{j}(k)^{\dagger} r_{j}(k)+ \\
+\sum_{l \in I_{N}} \sum_{s= \pm} \int d k \epsilon(k)\left(B_{s, l}^{\dagger}(k) B_{s, l}(k)+C_{s, l}^{\dagger}(k) C_{s, l}(k)\right)
\end{gathered}
$$

and

$$
\begin{aligned}
& H_{I}=\sum_{j=0}^{n-1}\left(r_{j}^{\dagger}\left(\bar{g}_{j}\right) a+r_{j}\left(g_{j}\right) a_{j}^{\dagger}\right)+\lambda \sum_{l \in I_{N}}\left(\phi_{l}^{(N)} b_{+, l}^{\dagger} b_{-, l}+h . c .\right)+ \\
& +\sum_{l \in I_{N}} \sum_{s= \pm}\left[b_{s, l}^{\dagger}\left(B_{s, l}\left(g_{B s}\right)+C_{s, l}\left(g_{C s}\right)\right)+\left(B_{s, l}^{\dagger}\left(g_{B s}\right)+C_{s, l}^{\dagger}\left(g_{C s}\right)\right) b_{s, l}\right] .
\end{aligned}
$$

Here $g_{B \pm}$ and $g_{C \pm}$ are real function, and the $\left\{b_{ \pm, l}^{\sharp}\right\}$ satisfy the following CAR $\left\{b_{ \pm, l}, b_{ \pm, l}^{\dagger}\right\}=1$ and they commutes when localized at different lattice sites: $\left[b_{ \pm, l}, b_{ \pm, s}^{\dagger}\right]=0$ if $l \neq s$. .

The commutation rules for the radiation operators (system and reservoir) coincide with the ones of the HL model. For what concerns the matter operators (system and reservoirs) the first remark is that any two operators localized at different lattice sites commutes, as well as any operator of the radiation with any observable of the matter. As for operators localized at the same lattice site, the only non trivial anticommutators are

$$
\left\{B_{ \pm, l}(k), B_{ \pm, l}^{\dagger}\left(k^{\prime}\right)\right\}=\left\{C_{ \pm, l}(k), C_{ \pm, l}^{\dagger}\left(k^{\prime}\right)\right\}=\delta\left(k-k^{\prime}\right)
$$


while all the others are zero. Finally, to clarify the different roles between the $B$ and the $C$ fields it is enough to consider their action on the ground state of the reservoir $\varphi_{0}$ :

$$
r_{j}(k) \varphi_{0}=B_{ \pm, l}(k) \varphi_{0}=C_{ \pm, l}^{\dagger}(k) \varphi_{0}=0 .
$$

These equations, together with what has been discussed, for instance, in [17], show that $B$ is responsible for the dissipation, while $C$ is the pump.

\section{III.1 Alli-Sewell versus Hepp-Lieb}

We begin this subsection with a pedagogical note on the single-mode single-atom version of the AS model. This will be useful in order to show that two reservoirs must be introduced to deal conveniently with the matter. After that we will consider the full AS model and we will show that the hamiltonian which produces the AS generator after considering its SL is nothing but the HL hamiltonian in the RWA. We will finally comment that adding the counter-rotating term (the one proportional to $\beta$ in (3.12)) does not affect this result, since its contribution disappear rigorously after the SL.

The starting point is given by the set of equations (3.3)-(3.10) restricted to $n=1$ and $N=0$, which means only one mode of radiation and a single atom. With this choice the phases in $\phi_{l}^{(N)}$ disappear so that the interaction hamiltonian (3.9) reduces to

$$
H_{\mathrm{int}}=i\left(\sigma_{-} a^{\dagger}-h . c .\right),
$$

and the total generator is $L=L_{\mathrm{mat}}+L_{\mathrm{rad}}+i\left[H_{\mathrm{int}},.\right]$.

Let us suppose that the atom is coupled not only to the radiation by means of $H_{\text {int }}$, but also to a bosonic background $m(k)$ with the easiest possible dipolar interaction:

$$
H_{M m}=\sigma_{+} m(h)+h . c .
$$

Of course this background must have a free dynamics and the natural choice is

$$
H_{0, m}=\int d k \omega_{m}(k) m^{\dagger}(k) m(k) .
$$

For what concerns the radiation background the situation is completely analogous:

$$
H_{0, r}=\int d k \omega_{r}(k) r^{\dagger}(k) r(k), \quad \quad H_{R, r}=a r^{\dagger}(\bar{g})+h . c .
$$


are respectively the free hamiltonian and the radiation-reservoir interaction. We take the complete hamiltonian as simply the sum of all these contributions, with the coupling constant $\lambda$ introduced as below:

$$
\begin{aligned}
& H=H_{0}+\lambda H_{I}=\left\{\mu \sigma_{z}+\omega_{R} a^{\dagger} a+\int d k \omega_{m}(k) m^{\dagger}(k) m(k)+\int d k \omega_{r}(k) r^{\dagger}(k) r(k)\right\}+ \\
& +\lambda\left\{\left(a r^{\dagger}(\bar{g})+\text { h.c. }\right)+\left(\sigma_{+} m(h)+\text { h.c. }\right)+\lambda i\left(\sigma_{-} a^{\dagger}-h . c .\right)\right\} .
\end{aligned}
$$

Taking the SL of this model simply means, first of all, considering the free evolution of the interaction hamiltonian, $H_{I}(t)=e^{i H_{0} t} H_{I} e^{-i H_{0} t}$. It is a simple computation to obtain that, if $\omega_{R}=2 \mu$, then

$$
H_{I}(t)=\left(a r^{\dagger}\left(\bar{g} e^{i\left(\omega_{r}-\omega_{R}\right) t}\right)+\text { h.c. }\right)+\left(\sigma_{+} m\left(h e^{i\left(2 \mu-\omega_{m}\right) t}\right)+h . c .\right)+\lambda i\left(\sigma_{-} a^{\dagger}-\text { h.c. }\right) .
$$

In this case the SL produces, as discussed in detail in [4], the following effective time-depending interaction hamiltonian:

$$
H_{I}^{(s l)}(t)=\left(\operatorname{ar}_{g}^{\dagger}(t)+h . c .\right)+\left(\sigma_{+} m_{h}(t)+h . c .\right)+i\left(\sigma_{-} a^{\dagger}-h . c .\right)
$$

where the dependence on $\lambda$ disappears and the operators $r_{g}(t), m_{h}(t)$ and their hermitian conjugates satisfy the following commutation relations for $t>t^{\prime}$,

$$
\left[r_{g}(t), r_{g}^{\dagger}\left(t^{\prime}\right)\right]=\Gamma_{-}^{(g)} \delta\left(t-t^{\prime}\right), \quad\left[m_{h}(t), m_{h}^{\dagger}\left(t^{\prime}\right)\right]=\Gamma_{-}^{(h)} \delta\left(t-t^{\prime}\right)
$$

Here we have defined the following complex quantities:

$$
\Gamma_{-}^{(g)}=\int_{-\infty}^{0} d \tau \int d k|g(k)|^{2} e^{-i\left(\omega_{r}(k)-\omega_{R}\right) \tau}, \quad \Gamma_{-}^{(h)}=\int_{-\infty}^{0} d \tau \int d k|h(k)|^{2} e^{-i\left(2 \mu-\omega_{m}(k)\right) \tau} .
$$

We want to stress that the restriction $t>t^{\prime}$ does not prevent to deduce the commutation rules (3.36) below, which are the main ingredient to compute the SL. However, the extension to $t<t^{\prime}$ can be easily obtained as discussed in [1]. Of course the functions $h$ and $g$ must be chosen in a such way that the integrals above exist finite!

In order to obtain the generator of the model we introduce the wave operator $U_{t}$ (in the interaction representation) which satisfies the following operator differential equation:

$$
\partial_{t} U_{t}=-i H_{I}^{(s l)}(t) U_{t}, \text { with } \quad U_{0}=\mathbb{1}
$$

We have already commented in Section II that, at least for a large class of quantum mechanical models, the equation above can be obtained as a suitable limit of differential equations for a 
$\lambda$-depending wave operator, [1]. Analogously, $r_{g}(t)$ and $m_{h}(t)$ can be considered as the limit (in the sense of the correlators) of the rescaled operators $\frac{1}{\lambda} r\left(g e^{-i\left(\omega_{r}-\omega_{R}\right) t / \lambda^{2}}\right)$ and $\frac{1}{\lambda} m\left(h e^{i\left(2 \mu-\omega_{m}\right) t / \lambda^{2}}\right)$. It is not surprising, therefore, that not only the operators but also the vectors of the Hilbert space of the theory are affected by the limiting procedure $\lambda \rightarrow 0$. In particular, the vacuum $\eta_{0}$ for the operators $r_{g}$ and $m_{h}, m_{h}(t) \eta_{0}=r_{g}(t) \eta_{0}=0$, does not coincide with the vacuum $\varphi_{0}$ for $m(k)$ and $r(k), r(k) \varphi_{0}=m(k) \varphi_{0}=0$, see [1] for more details.

Equation (3.34) above can be rewritten in the more convenient form

$$
U_{t}=\mathbb{1}-i \int_{0}^{t} H_{I}^{s l}\left(t^{\prime}\right) U_{t^{\prime}} d t^{\prime}
$$

which is used, together with the time consecutive principle, [1], to obtain the following useful commutation rules

$$
\left[r_{g}(t), U_{t}\right]=-i \Gamma_{-}^{(g)} a U_{t}, \quad\left[m_{h}(t), U_{t}\right]=-i \Gamma_{-}^{(h)} \sigma_{-} U_{t} .
$$

If we define the flow of a given observabe $X$ of the system as $j_{t}(X)=U_{t}^{\dagger}\left(X \otimes \mathbb{1}_{R}\right) U_{t}$, the generator is simply obtained by considering the expectation value of $\partial_{t} j_{t}(X)$ on a vector state $\eta_{0}^{(\xi)}=\eta_{0} \otimes \xi$, where $\xi$ is a generic state of the system. Using formulas (3.34), (3.36) and their hermitian conjugates, together with the properties of the vacuum $\eta_{0}$, the expression for the generator follows by identifying $L$ in the equation $\left\langle\partial_{t} j_{t}(X)\right\rangle_{\eta_{0}^{(\xi)}}=\left\langle j_{t}(L(X))\right\rangle_{\eta_{0}^{(\xi)}}$. The result is

$$
\begin{aligned}
& L(X)=L_{1}(X)+L_{2}(X)+L_{3}(X), \\
& L_{1}(X)=\Gamma_{-}^{(g)}\left[a^{\dagger}, X\right] a-\bar{\Gamma}_{-}^{(g)} a^{\dagger}[a, X], \quad L_{2}(X)=\Gamma_{-}^{(h)}\left[\sigma_{+}, X\right] \sigma_{-}-\bar{\Gamma}_{-}^{(h)} \sigma_{+}\left[\sigma_{-}, X\right], \\
& L_{3}(X)=i^{2}\left[\sigma_{-} a^{\dagger}-\sigma_{+} a, X\right]
\end{aligned}
$$

It is evident that both $L_{1}$ and $L_{3}$ can be rewritten in the same form of the radiation and interaction terms of the AS generator but this is not so, in general, for $L_{2}$ which has the form of the AS matter generator only if the pumping parameter $\eta$ is equal to -1 .

This is not very satisfactory and, how we will show in the following, is a consequence of having introduced a single reservoir for the atom. We will show that the existence of a second reservoir allows for the removal of the constraint $\eta=-1$ above.

With all of this in mind it is not difficult to produce an hamiltonian which should produce the full AS generator for the physical system with $2 N+1$ atoms and $n$ modes of radiation. With respect to the one discussed above, it is enough to double the number of reservoirs for the matter and to sum over $l \in I_{N}$ for the matter and over $j=0,1, \ldots, n-1$ for the modes. The resulting hamiltonian is therefore necessarely very close to the HL one:

$$
H=H_{0}+\lambda H_{I},
$$


with

$$
H_{0}=\omega_{R} \sum_{j=0}^{n-1} a_{j}^{\dagger} a_{j}+\mu \sum_{l \in I_{N}} \sigma_{l, z}+\sum_{l \in I_{N}} \sum_{s=1}^{2} \int d k \omega_{m_{s}}(k) m_{s, l}^{\dagger}(k) m_{s, l}(k)+\sum_{j=0}^{n-1} \int d k \omega_{r, j}(k) r_{j}(k)^{\dagger} r_{j}(k)
$$

and

$$
\begin{gathered}
H_{I}=\sum_{j=0}^{n-1}\left(r_{j}^{\dagger}\left(\bar{g}_{j}\right) a_{j}+r_{j}\left(g_{j}\right) a_{j}^{\dagger}\right)+\sum_{l \in I_{N}}\left[\left(m_{1, l}^{\dagger}\left(\bar{h}_{1}\right) \sigma_{l,-}+h . c .\right)+\left(m_{2, l}^{\dagger}\left(\bar{h}_{2}\right) \sigma_{l,+}+\text { h.c. }\right)\right]+ \\
+\lambda \sum_{l \in I_{N}}\left(\phi_{l}^{(N)} \sigma_{l,+}+\text { h.c. }\right)
\end{gathered}
$$

where the radiation field has been introduced in (3.10). It is clear that, but for the RWA which we are assuming here, there are not many other differences between this hamiltonian and the one in (3.11)-(3.15). It is worth mentioning that $\lambda$ appears both as an overall coupling constant, see (3.38), and as a multiplying factor of $\sum_{l \in I_{N}}\left(\phi_{l}^{(N)} \sigma_{l,+}+\right.$ h.c.) and plays the same role as $\sqrt{\alpha}$ in the HL hamiltonian. As for the commutation rules they are quite natural: but for the spin operators, which satisfy their own algebra, all the others operators satisfy the CCR and commute whenever they refer to different subsystems. In particular, for instance, all the $m_{1, l}^{\sharp}(k)$ commute with all the $m_{2, l^{\prime}}^{\sharp}\left(k^{\prime}\right)$, for all $k, k^{\prime}$ and $l, l^{\prime}$.

The procedure to obtain the generator is the same as before: we first compute $H_{I}(t)=$ $e^{i H_{0} t} H_{I} e^{-i H_{0} t}$, which enters in the differential equation for the wave operator. Taking the limit $\lambda \rightarrow 0$ of the mean value in the vector state defined by $\varphi_{0}^{(\xi)}=\varphi_{0} \otimes \xi$ of the first non trivial approximation of the rescaled version of $U_{t}$ we deduce the form of an effective hamiltonian, $H_{I}^{(s l)}(t)$, which is simply

$H_{I}^{(s l)}(t)=\sum_{j=0}^{n-1}\left(a_{j} r_{g, j}^{\dagger}(t)+h . c.\right)+\sum_{l \in I_{N}}\left(\sigma_{l,+} m_{1, l}(t)+h . c.\right)+\sum_{l \in I_{N}}\left(\sigma_{l,-} m_{2, l}(t)+h . c.\right)+\sum_{l \in I_{N}}\left(\phi_{l}^{(N)} \sigma_{l,+}+\right.$ h.c. $)$.

Again, we are assuming that $\omega_{R}=2 \mu$, which is crucial in order not to have a time dependence in the last term of $H_{I}^{(s l)}(t)$ in (3.41).

The only non trivial commutation rules for $t>t^{\prime}$ for the new operators are:

$$
\begin{aligned}
& {\left[r_{g, j}(t), r_{g, j^{\prime}}^{\dagger}\left(t^{\prime}\right)\right]=\Gamma_{-, j}^{(g)} \delta_{j, j^{\prime}} \delta\left(t-t^{\prime}\right),} \\
& {\left[m_{1, l}(t), m_{1, l^{\prime}}^{\dagger}\left(t^{\prime}\right)\right]=\Gamma_{-}^{\left(h_{1}\right)} \delta_{l, l^{\prime}} \delta\left(t-t^{\prime}\right),} \\
& {\left[m_{2, l}(t), m_{2, l^{\prime}}^{\dagger}\left(t^{\prime}\right)\right]=\Gamma_{-}^{\left(h_{2}\right)} \delta_{l, l^{\prime}} \delta\left(t-t^{\prime}\right),}
\end{aligned}
$$


where we have defined the following complex quantities:

$$
\begin{aligned}
\Gamma_{-, j}^{(g)} & =\int_{-\infty}^{0} d \tau \int d k\left|g_{j}(k)\right|^{2} e^{i\left(\omega_{r, j}(k)-\omega_{R}\right) \tau}, \quad \Gamma_{-}^{\left(h_{1}\right)}=\int_{-\infty}^{0} d \tau \int d k\left|h_{1}(k)\right|^{2} e^{i\left(\omega_{m_{1}}(k)-\omega_{R}\right) \tau}, \\
\Gamma_{-}^{\left(h_{2}\right)} & =\int_{-\infty}^{0} d \tau \int d k\left|h_{2}(k)\right|^{2} e^{i\left(\omega_{m_{2}}(k)+\omega_{R}\right) \tau} .
\end{aligned}
$$

From the commutation rules above and since $U_{t}=\mathbb{1}-i \int H_{I}^{(s l)}\left(t^{\prime}\right) U_{t^{\prime}} d t^{\prime}$, we get

$$
\left[r_{g, j}(t), U_{t}\right]=-i \Gamma_{-, j}^{(g)} a_{j} U_{t}, \quad\left[m_{1, l}(t), U_{t}\right]=-i \Gamma_{-}^{\left(h_{1}\right)} \sigma_{l,-} U_{t}, \quad\left[m_{2, l}(t), U_{t}\right]=-i \Gamma_{-}^{\left(h_{2}\right)} \sigma_{l,+} U_{t}
$$

The expression for the generator can be obtained as for the $N=0, n=1$ model described before, that is computing the mean value $\left\langle\partial_{t} j_{t}(X)\right\rangle_{\eta_{0}^{(\xi)}}$. Here, as before, $j_{t}(X)$ is the flux of the system observable $X, j_{t}(X)=U_{t}^{\dagger}\left(X \otimes \mathbb{1}_{R}\right) U_{t}$, and $\eta_{0}$ is the vacuum of the operators $r_{g, j}(t)$ and $m_{s, l}(t), s=1,2$. The computation gives the following result, which slightly generalize the one in $(3.37)$ :

$$
\begin{aligned}
& L(X)=L_{1}(X)+L_{2}(X)+L_{3}(X), \\
& L_{1}(X)=\sum_{j=0}^{n-1}\left(\Gamma_{-, j}^{(g)}\left[a_{j}^{\dagger}, X\right] a_{j}-\bar{\Gamma}_{-, j}^{(g)} a_{j}^{\dagger}\left[a_{j}, X\right]\right), \\
& L_{2}(X)=\sum_{l \in I_{N}}\left(\Gamma_{-}^{\left(h_{1}\right)}\left[\sigma_{+, l}, X\right] \sigma_{-, l}-\bar{\Gamma}_{-}^{\left(h_{1}\right)} \sigma_{+, l}\left[\sigma_{-, l}, X\right]+\Gamma_{-}^{\left(h_{2}\right)}\left[\sigma_{-, l}, X\right] \sigma_{+, l}-\bar{\Gamma}_{-}^{\left(h_{2}\right)} \sigma_{-, l}\left[\sigma_{+, l}, X\right],\right. \\
& L_{3}(X)=i \sum_{l \in I_{N}}\left[\left(\phi_{l}^{(N)} \sigma_{+, l}+\text { h.c. }\right), X\right] .
\end{aligned}
$$

It is not difficult to compare this generator with the one proposed by AS, see formulas ((3.3)$(3.10))$, and the conclusion is that the two generators are exactly the same provided that the following equalities are satisfied:

$$
\begin{aligned}
& \Im \Gamma_{-, j}^{(g)}=\omega_{j}, \quad \Re \Gamma_{-, j}^{(g)}=k_{j}, \quad \Re\left(\Gamma_{-}^{\left(h_{1}\right)}+\Gamma_{-}^{\left(h_{2}\right)}\right)=\gamma_{1}, \quad \Im\left(\Gamma_{-}^{\left(h_{1}\right)}-\Gamma_{-}^{\left(h_{2}\right)}\right)=\epsilon \\
& \Re \Gamma_{-}^{\left(h_{1}\right)}=\frac{1}{4} \gamma_{2}(1-\eta), \quad \Re \Gamma_{-}^{\left(h_{2}\right)}=\frac{1}{4} \gamma_{2}(1+\eta) .
\end{aligned}
$$

Therefore, if we start with the HL hamiltonian, choosing the regularizing functions in such a way that the equalities (3.46) are satisfied, the SL produces a generator of the model which is exactly the one proposed in $[12,13]$, with the extra minor constraint $\gamma_{2}=2 \gamma_{1}$, which is a direct consequence of (3.46).

We conclude this subsection with a remark concerning the role of the RWA and its relation with the SL. In particular, this is a very good approximation after the SL is taken. To show 
why, we first notice that adding a counter-rotating term (extending the one in (3.18)) to the interaction hamiltonian $H_{I}$ in (3.40), considering the same coupling constant for both the rotating and the counter-rotating term $(\beta=\alpha)$, simply means to add to $H_{I}$ in $(3.40)$ a contribution like $\lambda \sum_{l \in I_{N}}\left(\phi_{l}^{(N)} \sigma_{l,-}+h . c.\right)$. While the rotating term, if $2 \mu=\omega_{R}$, does not evolve freely, the free time evolution of this other term is not trivial. However, the differences with respect to the previous situation all disappear rigorously after the SL, because these extra contributions to the mean value of the wave operator go to zero when $\lambda \rightarrow 0$, so that at the end the expression for the generator is unchanged. This allows us to conclude that the full HL hamiltonian is equivalent to the AS generator, where the equivalence relation is provided by the SL.

\section{III.2 The SL of the DHL model}

We now consider the SL of the DHL model and we will get the expression of the related generator showing that, under some conditions on the quantities defining the model, the equations of motion do not differ from the ones in AS. The free evolved interaction hamiltonian $H_{I}$ in $(3.22)$ is,

$$
\begin{aligned}
& H_{I}(t)=e^{i H_{0} t} H_{I} e^{-i H_{0} t}=\sum_{j=0}^{n-1}\left(a_{j} r_{j}^{\dagger}\left(\bar{g}_{j} e^{i\left(\omega_{r, j}-\omega_{R}\right) t}\right)+h . c .\right)+\lambda \sum_{l \in I_{N}}\left(\phi_{l}^{(N)} b_{+, l}^{\dagger} b_{-, l}+h . c .\right)+ \\
& +\sum_{l \in I_{N}}\left[b_{+, l}^{\dagger}\left(B_{+, l}\left(g_{B+} e^{i t(\mu-\epsilon)}\right)+C_{+, l}\left(g_{C+} e^{i t(\mu-\epsilon)}\right)\right)+\left(B_{+, l}^{\dagger}\left(g_{B+} e^{-i t(\mu-\epsilon)}\right)+C_{+, l}^{\dagger}\left(g_{C+} e^{-i t(\mu-\epsilon)}\right)\right) b_{+, l}+\right. \\
& \left.+b_{-, l}^{\dagger}\left(B_{-, l}\left(g_{B-} e^{-i t(\mu+\epsilon)}\right)+C_{-, l}\left(g_{C-} e^{-i t(\mu+\epsilon)}\right)\right)+\left(B_{-, l}^{\dagger}\left(g_{B-} e^{i t(\mu+\epsilon)}\right)+C_{-, l}^{\dagger}\left(g_{C-} e^{i t(\mu+\epsilon)}\right)\right) b_{-, l}\right] .(3.47)
\end{aligned}
$$

Following the usual strategy we conclude that (the rescaled version of) the wave operator $U_{\lambda}(t)=\mathbb{1}-i \lambda \int_{0}^{t} H_{I}\left(t^{\prime}\right) U_{\lambda}\left(t^{\prime}\right) d t^{\prime}$ converges for $\lambda \rightarrow 0$ to another operator, which we still call a wave operator, satisfying the equation

$$
U_{t}=\mathbb{1}-i \int_{0}^{t} H_{I}^{(l s)}\left(t^{\prime}\right) U_{t^{\prime}} d t^{\prime}, \text { or, equivalently } \partial_{t} U_{t}=-i H_{I}^{(l s)}(t) U_{t}, \text { with } U_{0}=\mathbb{1} .
$$

Here $H_{I}^{(l s)}(t)$ is an effective time dependent hamiltonian, found using the usual strategy, defined as

$$
\begin{aligned}
H_{I}^{(l s)}(t)= & \sum_{j=0}^{n-1}\left(a_{j} r_{g, j}^{\dagger}(t)+\text { h.c. }\right)+\sum_{l \in I_{N}}\left(\phi_{l}^{(N)} b_{+, l}^{\dagger} b_{-, l}+h . c .\right)+\sum_{l \in I_{N}}\left[b_{+, l}^{\dagger}\left(\beta_{+, l}(t)+\gamma_{+, l}(t)\right)+\right. \\
& \left.+\left(\beta_{+, l}^{\dagger}(t)+\gamma_{+, l}^{\dagger}(t)\right) b_{+, l}+b_{-, l}^{\dagger}\left(\beta_{-, l}(t)+\gamma_{-, l}(t)\right)+\left(\beta_{-, l}^{\dagger}(t)+\gamma_{-, l}^{\dagger}(t)\right) b_{-, l}\right] .
\end{aligned}
$$

The operators of the reservoir which appear in $H_{I}^{(l s)}$ are the stochastic limit of the original (rescaled) time evoluted operators of the reservoir and satisfy (anti-)commutation relations 
which are related to those of the original ones. In particular, after the SL, any two operators of the matter (system and reservoirs) localized at different lattice sites commutes, as well as any operator of the radiation with any observable of the matter. As for operators localized at the same lattice site, the only non trivial anticommutators are

$$
\left\{\beta_{ \pm, l}(t), \beta_{ \pm, l}^{\dagger}\left(t^{\prime}\right)\right\}=\delta\left(t-t^{\prime}\right) \Gamma_{-}^{(B \pm)}, \quad\left\{\gamma_{ \pm, l}(t), \gamma_{ \pm, l}^{\dagger}\left(t^{\prime}\right)\right\}=\delta\left(t-t^{\prime}\right) \Gamma_{-}^{(C \pm)},
$$

which should be added to

$$
\left[r_{g, j}(t), r_{g, j^{\prime}}\left(t^{\prime}\right)\right]=\delta_{j, j^{\prime}} \delta\left(t-t^{\prime}\right) \Gamma_{-, j}^{(g)}
$$

In all these formulas the time ordering $t>t^{\prime}$ has to be understood and the following quantities are defined:

$$
\begin{aligned}
& \Gamma_{-, j}^{(g)}=\int_{-\infty}^{0} d \tau \int d k\left|g_{j}(k)\right|^{2} e^{i\left(\omega_{r, j}(k)-\omega_{R}\right) \tau}, \quad \Gamma_{-}^{(B \pm)}=\int_{-\infty}^{0} d \tau \int d k\left(g_{B \pm}(k)\right)^{2} e^{i(\epsilon(k) \mp \mu) \tau}, \\
& \Gamma_{-}^{(C \pm)}=\int_{-\infty}^{0} d \tau \int d k\left(g_{C \pm}(k)\right)^{2} e^{-i(\epsilon(k) \mp \mu) \tau} .
\end{aligned}
$$

We call now $\eta_{0}$ the vacuum of these limiting operators. We have

$$
r_{g, j}(t) \eta_{0}=\beta_{ \pm, l}(t) \eta_{0}=\gamma_{ \pm, l}^{\dagger}(t) \eta_{0}=0 .
$$

Paying a little attention to the fact that here commutators and anti-commutators simultaneously appear, we can compute the commutators between the operators $r_{g, j}(t)^{\sharp}, \gamma_{ \pm, l}^{\sharp}(t), \beta_{ \pm, l}^{\sharp}(t)$ and the wave operator $U_{t}$ by making use of $(3.50,3.51)$. We give here only those commutation rules which are used in the computation of the generator:

$$
\left[r_{g, j}(t), U_{t}\right]=-i \Gamma_{-, j}^{(g)} a_{j} U_{t}, \quad\left[\beta_{ \pm, l}(t), U_{t}\right]=-i \Gamma_{-}^{(B \pm)} b_{ \pm, l} U_{t}, \quad\left[\gamma_{ \pm, l}^{\dagger}(t), U_{t}\right]=i \Gamma_{-}^{(C \pm)} b_{ \pm, l}^{\dagger} U_{t} .
$$

Using the usual strategy, and restricting to quadratic matter operators for technical reasons (this condition can be avoided), [4], we get

$$
\begin{aligned}
L(X) & =L_{1}(X)+L_{2}(X)+L_{3}(X), \\
L_{1}(X) & =\sum_{j=0}^{n-1}\left(\Gamma_{-, j}^{(g)}\left[a_{j}^{\dagger}, X\right] a_{j}-\bar{\Gamma}_{-, j}^{(g)} a_{j}^{\dagger}\left[a_{j}, X\right]\right), \\
L_{2}(X) & =\sum_{l \in I_{N}}\left(\Gamma_{-}^{(B+)}\left[b_{+, l}^{\dagger}, X\right] b_{+, l}-\bar{\Gamma}_{-}^{(B+)} b_{+, l}^{\dagger}\left[b_{+, l}, X\right]+\Gamma_{-}^{(C+)}\left[b_{+, l}, X\right] b_{+, l}^{\dagger}-\bar{\Gamma}_{-}^{(C+)} b_{+, l}\left[b_{+, l}^{\dagger}, X\right]+\right. \\
+ & \left.\Gamma_{-}^{(B-)}\left[b_{-, l}^{\dagger}, X\right] b_{-, l}-\bar{\Gamma}_{-}^{(B-)} b_{-, l}^{\dagger}\left[b_{-, l}, X\right]+\Gamma_{-}^{(C-)}\left[b_{-, l}, X\right] b_{-, l}^{\dagger}-\bar{\Gamma}_{-}^{(C-)} b_{-, l}\left[b_{-, l}^{\dagger}, X\right]\right), \\
L_{3}(X) & =i \sum_{l \in I_{N}}\left[\left(\phi_{l}^{(N)} b_{+, l}^{\dagger} b_{-, l}+\text { h.c. }\right), X\right] .
\end{aligned}
$$


We see that the first and the last terms exactly coincide with the analogous contributions of the AS generator, but for a purely formal difference which is due to the different matter variables which are used in the two models. The second contribution, on the other hand, cannot be easily compared with the free AS matter generator. What is convenient, and sufficient, to get full insight about $L_{2}$, is to compute its action on a basis of the local algebra, that is on $b_{+, l}^{\dagger} b_{-, l}$ $\left(\equiv \sigma_{+, l}\right)$ and on $b_{+, l}^{\dagger} b_{+, l}-b_{-, l}^{\dagger} b_{-, l}\left(\equiv \sigma_{z, l}\right)$, all the others being trivial or an easy consequence of these ones. It is not hard to find the result:

$$
\begin{aligned}
& L_{2}\left(b_{+, l}^{\dagger} b_{-, l}\right)=-b_{+, l}^{\dagger} b_{-, l}\left(\Re\left[\Gamma_{-}^{(B+)}+\Gamma_{-}^{(B-)}+\Gamma_{-}^{(C+)}+\Gamma_{-}^{(C-)}\right]-\right. \\
& \left.-i \Im\left[\Gamma_{-}^{(B+)}-\Gamma_{-}^{(B-)}-\Gamma_{-}^{(C+)}+\Gamma_{-}^{(C-)}\right]\right), \\
& L_{2}\left(b_{+, l}^{\dagger} b_{+, l}-b_{-, l}^{\dagger} b_{-, l}\right)=2\left(-b_{+, l}^{\dagger} b_{+, l}\left(\Re\left[\Gamma_{-}^{(B+)}+\Gamma_{-}^{(C+)}\right)+\Re \Gamma_{-}^{(C+)}+\right.\right. \\
& +b_{-, l}^{\dagger} b_{-, l}\left(\Re\left[\Gamma_{-}^{(B-)}+\Gamma_{-}^{(C-)}\right)-\Re \Gamma_{-}^{(C-)}\right) .
\end{aligned}
$$

The equation for $\sigma_{+, l}$ is recovered without any problem, modulo some identifications $\left(\Re\left[\Gamma_{-}^{(B+)}+\right.\right.$ $\left.\left.\Gamma_{-}^{(B-)}+\Gamma_{-}^{(C+)}+\Gamma_{-}^{(C-)}\right]=\gamma_{1}, \ldots\right)$, while to recover the equation for $\sigma_{z, l}$ it is necessary to choose properly the regularizing functions which define the different $\Gamma_{-}$. In particular we need to have the following equality fulfilled:

$$
\Re\left(\Gamma_{-}^{(B+)}+\Gamma_{-}^{(C+)}\right)=\Re\left(\Gamma_{-}^{(B-)}+\Gamma_{-}^{(C-)}\right) .
$$

Under this condition we can conclude that the SL of the DHL model produces the same differential equations as the AS generator, as already happened for the HL model. It is also easy to check that, as a consequence of our approach, we must have $\gamma_{1}=\gamma_{2}$ in the generator we obtain. Of course this result is not surprising since already in the HL paper, [16], the fact that the two models are quite close (under some aspects) was pointed out. Here we have learned also that the SL of both these models, at least under some conditions, give rise to the same dynamical behavior.

\section{The open BCS model}

In this section we review some results on the Open BCS model, originally introduced in $[5,17]$, obtained using the SLA, [6].

The main outcome is that the same values of the critical temperature and of the order parameters can be found using the SLA, in a significantly simpler way. This simplification allows us to focus our attention on some aspects of the model which could appear not so clearly using the original approach. 


\section{IV.1 The Physicals Model and its stochastic limit}

Our model consists of two main ingredients, the system, which is described by spin variables, and the reservoir, which is given in terms of bosonic operators. We refer to Section III for the definition of the relevant operator algebras. The system is contained in a box of volume $V=L^{3}$, with $N$ lattice sites. We define, following $[5,17]$

$$
H_{N}^{(s y s)}=\tilde{\epsilon} \sum_{j=1}^{N} \sigma_{j}^{0}-\frac{g}{N} \sum_{i, j=1}^{N} \sigma_{i}^{+} \sigma_{j}^{-}
$$

where the indexes $i, j$ represent the discrete values of the momentum that an electron in a fixed volume can have, $\sigma_{j}^{+}$creates a Cooper pair with given momentum while $\sigma_{j}^{-}$annihilates the same pair, $\tilde{\epsilon}$ is the energy of a single electron and $-g<0$ is the interaction close to the Fermi surface. As we can see, only the \pm component of the spin, that is the $x, y$ components, have a mean field interaction, while the $z$ component interacts with a constant external magnetic field. The algebra of the Pauli matrices is given by

$$
\left[\sigma_{i}^{+}, \sigma_{j}^{-}\right]=\delta_{i j} \sigma_{i}^{0}, \quad\left[\sigma_{i}^{ \pm}, \sigma_{j}^{0}\right]=\mp 2 \delta_{i j} \sigma_{i}^{ \pm}
$$

We will use the following realization of these matrices:

$$
\sigma^{0} \equiv \sigma^{z}=\left(\begin{array}{cc}
1 & 0 \\
0 & -1
\end{array}\right), \quad \sigma^{+}=\left(\begin{array}{cc}
0 & 1 \\
0 & 0
\end{array}\right), \quad \sigma^{-}=\left(\begin{array}{cc}
0 & 0 \\
1 & 0
\end{array}\right) .
$$

If we now define the following operators,

$$
S_{N}^{\alpha}=\frac{1}{N} \sum_{i=1}^{N} \sigma_{i}^{\alpha}, \quad R_{N}=S_{N}^{+} S_{N}^{-}=R_{N}^{\dagger}
$$

$H_{N}^{(s y s)}$ can be simply written as $H_{N}^{(s y s)}=N\left(\tilde{\epsilon} S_{N}^{0}-g R_{N}\right)$ and it is easy to check that the following commutation rules hold:

$$
\left[S_{N}^{0}, R_{N}\right]=\left[H_{N}^{(s y s)}, R_{N}\right]=\left[H_{N}^{(s y s)}, S_{N}^{0}\right]=0
$$

for any given $N>0$. It is also worth noticing that the intensive operators $S_{N}^{\alpha}$ are all bounded by 1 in the operator norm, and that the commutators $\left[S_{N}^{\alpha}, \sigma_{j}^{\beta}\right]$ go to zero in norm as $\frac{1}{N}$ when $N \rightarrow \infty$, for all $j, \alpha$ and $\beta$.

Our construction of the reservoir follows the same steps given in [17], but for the commutation rules. We introduce here as many bosonic modes $a_{\vec{p}, j}$ as lattice sites are present in $V$. 
This means that $j=1,2, \ldots, N \cdot \vec{p}$ is the value of the momentum of the $\mathrm{j}$-th boson which, if we impose periodic boundary condition on the wave functions, has necessarily the form $\vec{p}=\frac{2 \pi}{L} \vec{n}$, where $\vec{n}=\left(n_{1}, n_{2}, n_{3}\right)$ with $n_{j} \in \mathbb{Z}$. These operators satisfy the following CCR,

$$
\left[a_{\vec{p}, i}, a_{\vec{q}, j}\right]=\left[a_{\vec{p}, i}^{\dagger}, a_{\vec{q}, j}^{\dagger}\right]=0, \quad\left[a_{\vec{p}, i}, a_{\vec{q}, j}^{\dagger}\right]=\delta_{i j} \delta_{\vec{p} \vec{q}}
$$

and their free dynamics is given by

$$
H_{N}^{(r e s)}=\sum_{j=1}^{N} \sum_{\vec{p} \in \Lambda_{N}} \epsilon_{\vec{p}} a_{\vec{p}, j}^{\dagger} a_{\vec{p}, j}
$$

Here $\Lambda_{N}$ is the set of values which $\vec{p}$ may take, according to the previous remark: $\Lambda_{N}=\{\vec{p}=$ $\left.\frac{2 \pi}{L} \vec{n}, \vec{n} \in \mathbb{Z}^{3}\right\}$. It is useful to stress that the energy of the different bosons is clearly independent of the lattice site: $\epsilon_{\vec{p}}=\frac{\vec{p}^{2}}{2 m}=\frac{4 \pi^{2}\left(n_{1}^{2}+n_{2}^{2}+n_{3}^{2}\right)}{2 m L^{2}}$.

The interaction is given by

$$
H_{N}^{(I)}=\sum_{j=1}^{N}\left(\sigma_{j}^{+} a_{j}(f)+h . c .\right)
$$

where we have introduced $a_{j}(f)=\sum_{\vec{p} \in \Lambda_{N}} a_{\vec{p}, j} f(\vec{p}), f$ being a given test function which will be asked to satisfy some extra conditions, see equation (4.24) below and the related discussion.

The finite volume open system is now described by the following hamiltonian,

$$
H_{N}=H_{N}^{0}+\lambda H_{N}^{(I)}, \text { where } H_{N}^{0}=H_{N}^{(s y s)}+H_{N}^{(r e s)}
$$

and $\lambda$ is the coupling constant.

As usual, the first step in the SLA is the computation of the free evolution of the interaction hamiltonian:

$$
H_{N}^{(I)}(t)=e^{i H_{N}^{0} t} H_{N}^{(I)} e^{-i H_{N}^{0} t}=\sum_{j=1}^{N}\left(e^{i H_{N}^{(s y s)} t} \sigma_{j}^{+} e^{-i H_{N}^{(s y s)} t} e^{i H_{N}^{(r e s)} t} a_{j}(f) e^{-i H_{N}^{(r e s)} t}+\text { h.c. }\right) .
$$

The computation of the part of the reservoir is trivial and produces

$$
e^{i H_{N}^{(r e s)} t} a_{j}(f) e^{-i H_{N}^{(r e s)} t}=a_{j}\left(f e^{-i t \epsilon}\right)
$$

where $a_{j}\left(f e^{-i t \epsilon}\right)=\sum_{\vec{p} \in \Lambda_{N}} a_{\vec{p}, j} f(\vec{p}) e^{-i t \epsilon_{\vec{p}}}$. This is an easy consequence of the CCR (4.4). The free evolution of the spin operators is more difficult and its expression can be found in [5, 17], for instance, where it is shown how to obtain the time evolution in a semiclassical approximation, 
i.e., when the free time evolution of the intensive operators $S_{N}^{\alpha}$ are replaced by their limits in a suitable topology, [19] and [6].

The differential equations of motion for the spin variables are

$$
\left\{\begin{array}{l}
\frac{d \sigma_{j}^{+}(t)}{d t}=2 i \tilde{\epsilon} \sigma_{j}^{+}(t)+i g S_{N}^{+}(t) \sigma_{j}^{0}(t) \\
\frac{d \sigma_{j}^{0}(t)}{d t}=2 i g\left(\sigma_{j}^{+}(t) S_{N}^{-}(t)-\sigma_{j}^{-}(t) S_{N}^{+}(t)\right) .
\end{array}\right.
$$

where $\sigma_{j}^{\alpha}(t)=e^{i H_{N}^{(s y s)} t} \sigma_{j}^{\alpha} e^{-i H_{N}^{(s y s)} t}$ and $S_{N}^{\alpha}(t)=e^{i H_{N}^{(s y s)} t} S_{N}^{\alpha} e^{-i H_{N}^{(s y s)} t}=\frac{1}{N} \sum_{j=1}^{N} e^{i H_{N}^{(s y s)} t} \sigma_{j}^{\alpha} e^{-i H_{N}^{(s y s)} t}=$ $\frac{1}{N} \sum_{j=1}^{N} \sigma_{j}^{\alpha}(t)$.

Let us now call $S^{\alpha}=\mathcal{F}-$ strong $\lim _{N \rightarrow \infty} S_{N}^{\alpha}$, where $\mathcal{F}$ is a suitable family of vectors. The proof of the existence of this limit (together with all its powers) may be found in [19] and references therein. We can now take the sum over $j=1,2, \ldots, N$ of (both sides of) the equations in (4.9), divide the result by $N$, and then consider the $\mathcal{F}-$ strong $\lim _{N \rightarrow \infty}$ of the equations obtained in this way. We find that $\dot{S}^{0}(t)=0$ and $\dot{S}^{+}(t)=i\left(2 \tilde{\epsilon}+g S^{0}(t)\right) S^{+}(t)$. These equations can be easily solved: $S^{0}(t)=S^{0}=\left(S^{0}\right)^{\dagger}$ and $S^{+}(t)=S^{+} e^{i\left(2 \tilde{\epsilon}+g S^{0}\right) t}$. Of course $S^{-}(t)=\left(S^{+}(t)\right)^{\dagger}$. The system (4.9) becomes, if we replace $S_{N}^{\alpha}(t)$ with its $\mathcal{F}-$ strong limit $S^{\alpha}(t)$,

$$
\left\{\begin{array}{l}
\frac{d \sigma_{j}^{+}(t)}{d t}=2 i \tilde{\epsilon} \sigma_{j}^{+}(t)+i g S^{+}(t) \sigma_{j}^{0}(t) \\
\frac{d \sigma_{j}^{0}(t)}{d t}=2 i g\left(\sigma_{j}^{+}(t) S^{-}(t)-\sigma_{j}^{-}(t) S^{+}(t)\right) .
\end{array}\right.
$$

This system is called the semiclassical approximation of (4.9), and it can be explicitly solved:

$$
\sigma_{j}^{+}(t)=e^{i \nu t} \rho_{0}^{j}+e^{i(\nu+\omega) t} \rho_{+}^{j}+e^{i(\nu-\omega) t} \rho_{-}^{j},
$$

where we have defined the following operators

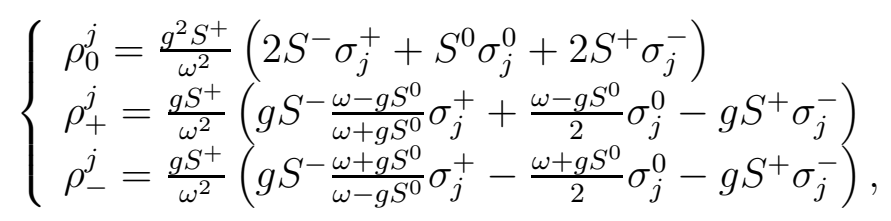

and the following quantities

$$
\omega=g \sqrt{\left(S^{0}\right)^{2}+4 S^{+} S^{-}}, \nu=2 \tilde{\epsilon}+g S^{0} .
$$

Defining further

$$
\nu_{\alpha}(\vec{p})=\nu-\epsilon_{\vec{p}}+\alpha \omega,
$$

where $\alpha$ takes the values $0,+$ and - , the operator $H_{N}^{(I)}(t)$ in $(4.8)$ becomes

$$
H_{N}^{(I)}(t)=\sum_{j=1}^{N} \sum_{\alpha=0, \pm}\left(\rho_{\alpha}^{j} a_{j}\left(f e^{i t \nu_{\alpha}}\right)+h . c\right) .
$$


The next step in the SLA consists in computing the following quantity

$$
I_{\lambda}(t)=\left(-\frac{i}{\lambda}\right)^{2} \int_{0}^{t} d t_{1} \int_{0}^{t_{1}} d t_{2} \omega_{t o t}\left(H_{N}^{(I)}\left(\frac{t_{1}}{\lambda^{2}}\right) H_{N}^{(I)}\left(\frac{t_{2}}{\lambda^{2}}\right)\right),
$$

and its limit for $\lambda$ going to zero. Here the state $\omega_{\text {tot }}$ is the following product state $\omega_{\text {tot }}=\omega_{\text {sys }} \omega_{\beta}$, where $\omega_{\text {sys }}$ is a generic state of the system, while $\omega_{\beta}$ is a state of the reservoir, which we will take to be a KMS state corresponding to an inverse temperature $\beta=\frac{1}{k T}$. It is convenient here to use the so-called canonical representation of thermal states, [1], which is sketched in the Appendix. Then we introduce two sets of mutually commuting bosonic operators $\left\{c_{\vec{p}, j}^{(\gamma)}\right\}$, $\gamma=a, b$, as follows:

$$
a_{\vec{p}, j}=\sqrt{m(\vec{p})} c_{\vec{p}, j}^{(a)}+\sqrt{n(\vec{p})} c_{\vec{p}, j}^{(b), \dagger}
$$

where

$$
m(\vec{p})=\omega_{\beta}\left(a_{\vec{p}, j} a_{\vec{p}, j}^{\dagger}\right)=\frac{1}{1-e^{-\beta \epsilon_{\vec{p}}}}, \quad n(\vec{p})=\omega_{\beta}\left(a_{\vec{p}, j}^{\dagger} a_{\vec{p}, j}\right)=\frac{e^{-\beta \epsilon_{\vec{p}}}}{1-e^{-\beta \epsilon_{\vec{p}}}} .
$$

The operators $c_{\vec{p}, j}^{(\alpha)}$ satisfy the following commutation rules

$$
\left[c_{\vec{p}, j}^{(\alpha)}, c_{\vec{q}, k}^{(\gamma)^{\dagger}}\right]=\delta_{j k} \delta_{\vec{p} \vec{q}} \delta_{\alpha \gamma},
$$

while all the other commutators are trivial. Furthermore, we introduce the vacuum of the operators $c_{\vec{p}, j}^{(\alpha)}, \Phi_{0}$ :

$$
c_{\vec{p}, j}^{(\alpha)} \Phi_{0}=0, \quad \forall \vec{p} \in \Lambda_{N}, j=1, . . N, \alpha=a, b .
$$

Finally, if we define $f_{m}(\vec{p})=\sqrt{m(\vec{p})} f(\vec{p})$ and $f_{n}(\vec{p})=\sqrt{n(\vec{p})} f(\vec{p})$, we get

$$
a_{j}\left(f e^{i t \nu_{\alpha}}\right)=c_{j}^{(a)}\left(f_{m} e^{i t \nu_{\alpha}}\right)+c_{j}^{(b)^{\dagger}}\left(f_{n} e^{i t \nu_{\alpha}}\right),
$$

using the usual notation for $c_{j}^{(a)}(g)$ and $c_{j}^{(b)^{\dagger}}(g)$. Therefore we have

$$
H_{N}^{(I)}(t)=\sum_{j=1}^{N} \sum_{\alpha=0, \pm}\left\{\rho_{\alpha}^{j}\left(c_{j}^{(a)}\left(f_{m} e^{i t \nu_{\alpha}}\right)+c_{j}^{(b)^{\dagger}}\left(f_{n} e^{i t \nu_{\alpha}}\right)\right)+h . c\right\}
$$

and the KMS state $\omega_{\beta}$ can be represented as the following vector state, as in a GNS-like representation:

$$
\omega_{\beta}\left(X_{r}\right)=\left\langle\Phi_{0}, X_{r} \Phi_{0}\right\rangle
$$

for any observable of the reservoir, $X_{r}$, since $\omega_{\beta}$ is a gaussian state, [1]. This fact, together with (4.20) and with the commutation rules (4.19), simplifies the computation of the two point function $\omega_{t o t}\left(H_{N}^{(I)}\left(\frac{t_{1}}{\lambda^{2}}\right) H_{N}^{(I)}\left(\frac{t_{2}}{\lambda^{2}}\right)\right)$, which produces

$$
\omega_{t o t}\left(H_{N}^{(I)}\left(\frac{t_{1}}{\lambda^{2}}\right) H_{N}^{(I)}\left(\frac{t_{2}}{\lambda^{2}}\right)\right)=\sum_{j=1}^{N} \sum_{\alpha, \beta=0, \pm \vec{p} \in \Lambda_{N}}\left\{\omega_{s y s}\left(\rho_{\alpha}^{j} \rho_{\beta}^{j \dagger}\right)\left|f_{m}(\vec{p})\right|^{2} e^{i \frac{t_{1}}{\lambda^{2}} \nu_{\alpha}(\vec{p})} e^{-i \frac{t_{2}}{\lambda^{2}} \nu_{\beta}(\vec{p})}+\right.
$$




$$
\left.+\omega_{\text {sys }}\left(\rho_{\alpha}^{j^{\dagger}} \rho_{\beta}^{j}\right)\left|f_{n}(\vec{p})\right|^{2} e^{-i \frac{t_{1}}{\lambda^{2}} \nu_{\alpha}(\vec{p})} e^{+i \frac{t_{2}}{\lambda^{2}} \nu_{\beta}(\vec{p})}\right\} .
$$

Since we are interested to the limit $\lambda \rightarrow 0$ of $I_{\lambda}(t)$ we need to impose some conditions on the test function $f(\vec{p})$, [1]. In particular, we will require that the following integral exists finite:

$$
\int_{-\infty}^{0} d \tau \sum_{\vec{p} \in \Lambda_{N}}\left|f_{r}(\vec{p})\right|^{2} e^{ \pm i \tau \nu_{\alpha}(\vec{p})}<\infty
$$

where $f_{r}(\vec{p})$ is $f_{m}(\vec{p})$ or $f_{n}(\vec{p})$ and $\nu_{\alpha}(\vec{p})$ is given in $(4.14)$. Under this assumption we find that

$$
I(t)=\lim _{\lambda \rightarrow 0} I_{\lambda}(t)=-t \sum_{j=1}^{N} \sum_{\alpha=0, \pm}\left\{\omega_{s y s}\left(\rho_{\alpha}^{j} \rho_{\alpha}^{j \dagger}\right) \Gamma_{\alpha}^{(a)}+\omega_{s y s}\left(\rho_{\alpha}^{j \dagger} \rho_{\alpha}^{j}\right) \Gamma_{\alpha}^{(b)}\right\},
$$

where the two complex quantities

$$
\Gamma_{\alpha}^{(a)}=\int_{-\infty}^{0} d \tau \sum_{\vec{p} \in \Lambda_{N}}\left|f_{m}(\vec{p})\right|^{2} e^{-i \tau \nu_{\alpha}(\vec{p})}, \quad \Gamma_{\alpha}^{(b)}=\int_{-\infty}^{0} d \tau \sum_{\vec{p} \in \Lambda_{N}}\left|f_{n}(\vec{p})\right|^{2} e^{i \tau \nu_{\alpha}(\vec{p})}
$$

both exist because of the assumption (4.24).

To this same result we could also arrive starting with the following stochastic limit hamiltonian

$$
H_{N}^{(s l)}(t)=\sum_{j=1}^{N} \sum_{\alpha=0, \pm}\left\{\rho_{\alpha}^{j}\left(c_{\alpha j}^{(a)}(t)+c_{\alpha j}^{(b)^{\dagger}}(t)\right)+h . c\right\},
$$

where the operators $c_{\alpha j}^{(\gamma)}(t)$ satisfy the following commutation rule,

$$
\left[c_{\alpha j}^{(\gamma)}(t), c_{\beta k}^{(\mu)^{\dagger}}\left(t^{\prime}\right)\right]=\delta_{j k} \delta_{\alpha \beta} \delta_{\gamma \mu} \delta\left(t-t^{\prime}\right) \Gamma_{\alpha}^{(\gamma)}, \quad \text { for } t>t^{\prime}
$$

We mean that, as it is easily checked, the following quantity

$$
J(t)=(-i)^{2} \int_{0}^{t} d t_{1} \int_{0}^{t_{1}} d t_{2} \Omega_{t o t}\left(H_{N}^{(s l)}\left(t_{1}\right) H_{N}^{(s l)}\left(t_{2}\right)\right)
$$

coincides with $I(t)$. Here $\Omega_{t o t}=\omega_{\text {sys }} \Omega=\omega_{\text {sys }}\left\langle\Psi_{0}, \Psi_{0}\right\rangle$, where $\Psi_{0}$ is the vacuum of the operators $c_{\alpha j}^{(\gamma)}(t): c_{\alpha j}^{(\gamma)}(t) \Psi_{0}=0$ for all $\alpha, j, \gamma$ and $t,[1]$.

We now use $H_{N}^{(s l)}(t)$ to compute the generator of the theory. Let $X$ be an observable of the system and $\mathbb{1}_{r}$ the identity of the reservoir. Its time evolution (after the stochastic limit is taken $)$ is $j_{t}\left(X \otimes \mathbb{1}_{r}\right)=U_{t}^{\dagger}\left(X \otimes \mathbb{1}_{r}\right) U_{t}$, where $U_{t}$ is the wave operator satisfying the following differential equation $\partial_{t} U_{t}=-i H_{N}^{(s l)}(t) U_{t}$, whose adjoint is $\partial_{t} U_{t}^{\dagger}=i U_{t}^{\dagger} H_{N}^{(s l)}(t)$.

Then we find

$$
\partial_{t} j_{t}\left(X \otimes \mathbb{1}_{r}\right)=i U_{t}^{\dagger}\left[H_{N}^{(s l)}(t), X \otimes \mathbb{1}_{r}\right] U_{t}=
$$




$$
=i U_{t}^{\dagger} \sum_{j=1}^{N} \sum_{\alpha=0, \pm}\left\{\left[\rho_{\alpha}^{j}, X\right]\left(c_{\alpha j}^{(a)}(t)+c_{\alpha j}^{(b)}(t)\right)+\left[\rho_{\alpha}^{j}{ }^{\dagger}, X\right]\left(c_{\alpha j}^{(a)^{\dagger}}(t)+c_{\alpha j}^{(b)}(t)\right)\right\} U_{t}
$$

Using now the commutation rules

$$
\left[c_{\alpha j}^{(a)}(t), U_{t}\right]=-i \int_{0}^{t}\left[c_{\alpha j}^{(a)}(t), H_{N}^{(s l)}\left(t^{\prime}\right)\right] U_{t^{\prime}} d t^{\prime}=-i \int_{0}^{t}\left(\rho_{\alpha}^{j \dagger} \Gamma_{\alpha}^{(a)} \delta\left(t-t^{\prime}\right)\right) U_{t^{\prime}} d t^{\prime}=-i \rho_{\alpha}^{j \dagger} \Gamma_{\alpha}^{(a)} U_{t}
$$

and

$$
\left[c_{\alpha j}^{(b)}(t), U_{t}\right]=-i \rho_{\alpha}^{j} \Gamma_{\alpha}^{(b)} U_{t}
$$

and their adjoints, we find that

$$
\begin{gathered}
\partial_{t} j_{t}\left(X \otimes \mathbb{1}_{r}\right)=i \sum_{j=1}^{N} \sum_{\alpha=0 \pm}\left\{\left(i U_{t}^{\dagger} \rho_{\alpha}^{j \dagger} \overline{\Gamma_{\alpha}^{(b)}}+c_{\alpha j}^{(b)}(t) U_{t}^{\dagger}\right)\left[\rho_{\alpha}^{j}, X\right] U_{t}+\right. \\
\left(i U_{t}^{\dagger} \rho_{\alpha}^{j} \overline{\Gamma_{\alpha}^{(a)}}+c_{\alpha j}^{(a)}(t) U_{t}^{\dagger}\right)\left[\rho_{\alpha}^{j \dagger}, X\right] U_{t}+U_{t}^{\dagger}\left[\rho_{\alpha}^{j}, X\right]\left(-i \rho_{\alpha}^{j \dagger} \Gamma_{\alpha}^{(a)} U_{t}+U_{t} c_{\alpha j}^{(a)}(t)\right)+ \\
\left.+U_{t}^{\dagger}\left[\rho_{\alpha}^{j \dagger}, X\right]\left(-i \rho_{\alpha}^{j} \Gamma_{\alpha}^{(b)} U_{t}+U_{t} c_{\alpha j}^{(b)}(t)\right)\right\}
\end{gathered}
$$

which has to be computed on the state $\Omega_{t o t}$. Therefore, since the generator $L$ satisfies the equality $\Omega_{t o t}\left(\partial_{t} j_{t}\left(X \otimes \mathbb{1}_{r}\right)\right)=\Omega_{t o t}\left(j_{t}(L(X))\right)$, we get

$$
L(X)=\sum_{j=1}^{N} \sum_{\alpha=0 \pm}\left\{\left[\rho_{\alpha}^{j}, X\right] \rho_{\alpha}^{j \dagger} \Gamma_{\alpha}^{(a)}+\left[\rho_{\alpha}^{j \dagger}, X\right] \rho_{\alpha}^{j} \Gamma_{\alpha}^{(b)}-\rho_{\alpha}^{j}\left[\rho_{\alpha}^{j \dagger}, X\right] \overline{\Gamma_{\alpha}^{(a)}}-\rho_{\alpha}^{j \dagger}\left[\rho_{\alpha}^{j}, X\right] \overline{\Gamma_{\alpha}^{(b)}}\right\}
$$

This expression can be made simpler if the observable $X$ is self-adjoint $\left(X=X^{\dagger}\right)$. In this case we have

$$
L(X)=L_{1}(X)+L_{2}(X)
$$

where

$$
L_{1}(X)=\sum_{j=1}^{N} \sum_{\alpha=0 \pm}\left\{\left[\rho_{\alpha}^{j}, X\right] \rho_{\alpha}^{j \dagger} \Gamma_{\alpha}^{(a)}+\text { h.c. }\right\}, \quad L_{2}(X)=\sum_{j=1}^{N} \sum_{\alpha=0 \pm}\left\{\left[\rho_{\alpha}^{j^{\dagger}}, X\right] \rho_{\alpha}^{j} \Gamma_{\alpha}^{(b)}+\text { h.c. }\right\} .
$$

\section{IV.2 The phase transition}

As discussed in [5, 17], $S_{N}^{0}$ and $R_{N}$ are the relevant variables whose dynamics must be considered to analyze the phase structure of the model. These intensive operators are both self-adjoint, so that we can use equations (4.32) and (4.33) instead of (4.31). As a matter of fact, in both [5] and [17] these equations of motion are considered only as an intermediate step to compute the equation for $\Delta_{N}=\frac{1}{2} R_{N}^{1 / 2}$, which is called the gap operator. We have shown in [6] that the 
same conclusions as in $[5,17]$ can be obtained without introducing $\Delta_{N}$ but working directly with $R_{N}$ and $S_{N}^{0}$.

First we focus on $L\left(S_{N}^{0}\right)=L_{1}\left(S_{N}^{0}\right)+L_{2}\left(S_{N}^{0}\right)$. We have, using (4.2), (4.3) and (4.33)

$$
L_{1}\left(S_{N}^{0}\right)=\frac{1}{N} \sum_{j=1}^{N} L_{1}\left(\sigma_{j}^{0}\right)=\frac{1}{N} \sum_{j=1}^{N} \sum_{\alpha=0, \pm}\left\{\left[\rho_{\alpha}^{j}, \sigma_{j}^{0}\right] \rho_{\alpha}^{j \dagger} \Gamma_{\alpha}^{(a)}+\text { h.c. }\right\}
$$

whose limit in the $\mathcal{F}-$ strong topology exists, [19], and is given by

$$
L_{1}\left(S^{0}\right):=\mathcal{F}-\text { strong } \lim _{N \rightarrow \infty} L_{1}\left(S_{N}^{0}\right)=-\frac{8 g^{4} S^{0}\left(S^{+} S^{-}\right)^{2}}{\omega^{3}}\left\{\Re \Gamma_{+}^{(a)} \frac{\omega-g}{\left(\omega+g S^{0}\right)^{2}}+\Re \Gamma_{-}^{(a)} \frac{\omega+g}{\left(\omega-g S^{0}\right)^{2}}\right\},
$$

where $\Re \Gamma_{ \pm}^{(a)}$ indicates the real part of $\Gamma_{ \pm}^{(a)},[6]$.

The computation of $L_{2}\left(S^{0}\right):=\mathcal{F}-$ strong $\lim _{N \rightarrow \infty} L_{2}\left(S_{N}^{0}\right)$ follows essentially the same steps and produces

$$
L_{2}\left(S^{0}\right)=-\frac{8 g^{4} S^{0}\left(S^{+} S^{-}\right)^{2}}{\omega^{3}}\left\{\Re \Gamma_{+}^{(b)} \frac{\omega+g}{\left(\omega+g S^{0}\right)^{2}}+\Re \Gamma_{-}^{(b)} \frac{\omega-g}{\left(\omega-g S^{0}\right)^{2}}\right\},
$$

[6], so that the final result is

$$
L\left(S^{0}\right)=-\frac{8 g^{4} S^{0}\left(S^{+} S^{-}\right)^{2}}{\omega^{3}} h\left(S^{0}, S^{+} S^{-}\right) .
$$

Here we have introduced, for brevity, the function

$$
h\left(S^{0}, S^{+} S^{-}\right)=\Re \Gamma_{+}^{(a)} \frac{\omega-g}{\left(\omega+g S^{0}\right)^{2}}+\Re \Gamma_{-}^{(a)} \frac{\omega+g}{\left(\omega-g S^{0}\right)^{2}}+\Re \Gamma_{+}^{(b)} \frac{\omega+g}{\left(\omega+g S^{0}\right)^{2}}+\Re \Gamma_{-}^{(b)} \frac{\omega-g}{\left(\omega-g S^{0}\right)^{2}},
$$

and we have written explicitly the dependence of $h$ on $S^{+} S^{-}=\mathcal{F}-$ strong $\lim _{N \rightarrow \infty} S_{N}^{+} S_{N}^{-}$, see (4.13). It is interesting to observe that the same function $h\left(S^{0}, S^{+} S^{-}\right)$appears in the computation of $L\left(S^{+} S^{-}\right):=\mathcal{F}-$ strong $\lim _{N \rightarrow \infty} L\left(S_{N}^{+} S_{N}^{-}\right)$. Again, since $\left(S_{N}^{+} S_{N}^{-}\right)^{\dagger}=S_{N}^{+} S_{N}^{-}$, we can use formulas (4.32) and (4.33). Here the computations are significantly harder, but no difficulty of principle arises. As a technical tool it is convenient to use the fact that, in the limit $N \rightarrow \infty$, all the intensive operators commute with all the local operators of the system, $\lim _{N \rightarrow \infty}\left[S_{N}^{\alpha}, \sigma_{j}^{\beta}\right]=0$, for all $\alpha, \beta$ and $j$. Therefore we get

$$
L\left(S^{+} S^{-}\right)=-\frac{16 g^{4}\left(S^{+} S^{-}\right)^{3}}{\omega^{3}} h\left(S^{0}, S^{+} S^{-}\right) .
$$

The phase structure of the model is now given by the right-hand sides of equations (4.36) and $(4.38)$, see $[5,17]$, and, in particular, from the zeros of the functions

$$
f_{1}(x, y)=-\frac{8 g^{4} x y^{2}}{\omega^{3}} h(x, y), \quad f_{2}(x, y)=-\frac{16 g^{4} y^{3}}{\omega^{3}} h(x, y)
$$


where we have introduced, to simplify the notation, $x=S^{0}$ and $y=S^{+} S^{-}$. In particular, the existence of a superconducting phase corresponds to the existence of a non trivial zero of $f_{1}$ and $f_{2},[5,17]$. Due to the definition of $f_{1}$ and $f_{2}$ it is clear that a pair $\left(x_{o}, y_{o}\right)$, with $x_{o} \neq 0$ and $y_{o} \neq 0$, is such that $f_{1}\left(x_{o}, y_{o}\right)=f_{2}\left(x_{o}, y_{o}\right)=0$ if and only if it is a zero of the function $h$ : $h\left(x_{o}, y_{o}\right)=0$. In order to find such a zero, it is first necessary to obtain an explicit expression for the coefficients $\Re \Gamma_{ \pm}^{(\gamma)}$. This is easily done using the definitions in (4.26), since we get

$$
\Re \Gamma_{ \pm}^{(a)}=\frac{1}{2} \int_{-\infty}^{\infty} \sum_{\vec{p} \in \Lambda_{N}}\left|f_{m}(\vec{p})\right|^{2} e^{-i \tau \nu_{ \pm}(\vec{p})} d \tau=\pi \sum_{\vec{p} \in \Lambda_{N}}\left|f_{m}(\vec{p})\right|^{2} \delta\left(\nu_{ \pm}(\vec{p})\right),
$$

and

$$
\Re \Gamma_{ \pm}^{(b)}=\pi \sum_{\vec{p} \in \Lambda_{N}}\left|f_{n}(\vec{p})\right|^{2} \delta\left(\nu_{ \pm}(\vec{p})\right)
$$

It is now almost straightforward to recover the results of $[5,17]$. Following Buffet and Martin's original idea, we look for solutions corresponding to $\nu=0$. This means that, because of (4.13), the value of $x=S^{0}$ is fixed: $x=-2 \tilde{\epsilon} / g$. Moreover, with this choice, $\nu_{+}(\vec{p})=\omega-\epsilon_{\vec{p}}$, which is zero if and only if $\omega=\epsilon_{\vec{p}}$. Also, we have $\nu_{-}(\vec{p})=-\omega-\epsilon_{\vec{p}}$, which is never zero. For these reasons we deduce that $\Re \Gamma_{-}^{(\gamma)}=0, \gamma=a, b$, while the sums in (4.40) and (4.41) for $\Re \Gamma_{+}^{(\gamma)}$ are restricted to the smaller set, $\mathcal{E}_{N} \subset \Lambda_{N}$, of those values of $\vec{p}$ such that, if $\vec{q} \in \mathcal{E}_{N}$ then $\epsilon_{\vec{q}}=\omega$. Therefore, recalling the expression of $m(\vec{p})$ and $n(\vec{p})$ in $(4.18)$, we find

$$
\Re \Gamma_{+}^{(a)}=\pi \frac{e^{\beta \omega}}{e^{\beta \omega}-1} \sum_{\vec{p} \in \mathcal{E}_{N}}|f(\vec{p})|^{2}, \quad \Re \Gamma_{+}^{(b)}=\pi \frac{1}{e^{\beta \omega}-1} \sum_{\vec{p} \in \mathcal{E}_{N}}|f(\vec{p})|^{2},
$$

so that equation $f(x, y)=0$ looks like

$$
\pi \frac{e^{\beta \omega}}{e^{\beta \omega}-1} \sum_{\vec{p} \in \mathcal{E}_{N}}|f(\vec{p})|^{2} \frac{\omega-g}{(\omega+g x)^{2}}+\pi \frac{1}{e^{\beta \omega}-1} \sum_{\vec{p} \in \mathcal{E}_{N}}|f(\vec{p})|^{2} \frac{\omega+g}{(\omega+g x)^{2}}=0,
$$

or

$$
e^{\beta \omega}=\frac{g+\omega}{g-\omega} .
$$

This equation is the crucial one, which replaces the one obtained in $[5,17], g \tanh \left(\frac{\beta \omega}{2}\right)=\omega$. As a matter of fact, in [6] it is also proven that these two equations are equivalent, and for that we recover exactly the same values of the critical temperature and of the order parameters as in $[5]$. 


\section{IV.3 More results}

We have shown how the SLA can be successfully used to analyze the phase structure of low temperature superconductivity analyzing a strong coupling BCS model, considered as an open system interacting with a bosonic thermal bath.

This procedure is rather direct and technically much simpler than the one used in [5]. Among the other simplifications, for instance, a single equation $h(x, y)=0$ must be solved instead of the system $f_{1}(x, y)=f_{2}(x, y)=0$. This suggests to use the SLA also to modify the original model in the attempt of getting some insight on high-temperature superconductivity. This project started quite recently, [7], by introducing two reservoirs instead of only one, as we did here, to see whether the value of the critical temperature increases because of the presence of this second reservoir. Our results seem rather promising but not jet definitive. A deeper analysis is being presently undertaken.

\section{Acknowledgments}

I would like to acknowledge financial support by the Murst, within the project Problemi Matematici Non Lineari di Propagazione e Stabilità nei Modelli del Continuo, coordinated by Prof. T. Ruggeri. 


\section{Appendix: Few results on the stochastic limit}

In this Appendix we will briefly summarize some of the basic facts and properties concerning the SLA which are used all throughout the paper. We refer to [1] and references therein for more details.

Given an open system $\mathcal{S}+\mathcal{R}$ we write its hamiltonian $H$ as the sum of two contributions, the free part $H_{0}$ and the interaction $\lambda H_{I}$. Here $\lambda$ is a coupling constant, $H_{0}$ contains the free evolution of both the system $\mathcal{S}$ and the reservoir $\mathcal{R}$, while $H_{I}$ contains the interaction between $\mathcal{S}$ and $\mathcal{R}$. Working in the interaction picture, we define $H_{I}(t)=e^{i H_{0} t} H_{I} e^{-i H_{0} t}$ and the so called wave operator $U_{\lambda}(t)$ which is the solution of the following differential equation

$$
\partial_{t} U_{\lambda}(t)=-i \lambda H_{I}(t) U_{\lambda}(t)
$$

with the initial condition $U_{\lambda}(0)=\mathbb{1}$. Using the van-Hove rescaling $t \rightarrow \frac{t}{\lambda^{2}}$, see $[17,1]$ for instance, we can rewrite the same equation in a form which is more convenient for our perturbative approach, that is

$$
\partial_{t} U_{\lambda}\left(\frac{t}{\lambda^{2}}\right)=-\frac{i}{\lambda} H_{I}\left(\frac{t}{\lambda^{2}}\right) U_{\lambda}\left(\frac{t}{\lambda^{2}}\right)
$$

with the same initial condition as before. Its integral counterpart is

$$
U_{\lambda}\left(\frac{t}{\lambda^{2}}\right)=\mathbb{1}-\frac{i}{\lambda} \int_{0}^{t} H_{I}\left(\frac{t^{\prime}}{\lambda^{2}}\right) U_{\lambda}\left(\frac{t^{\prime}}{\lambda^{2}}\right) d t^{\prime},
$$

which is the starting point for a perturbative expansion, which works in the following way.

Suppose, to begin with, that we are interested to the zero temperature situation. Then let $\varphi_{0}$ be the ground vector of the reservoir and $\xi$ a generic vector of the system. Now we put $\varphi_{0}^{(\xi)}=\varphi_{0} \otimes \xi$. We want to compute the limit, for $\lambda$ going to 0 , of the first non trivial order of the mean value of the perturbative expansion of $U_{\lambda}\left(t / \lambda^{2}\right)$ above in $\varphi_{0}^{(\xi)}$, that is the limit of

$$
I_{\lambda}(t)=\left(-\frac{i}{\lambda}\right)^{2} \int_{0}^{t} d t_{1} \int_{0}^{t_{1}} d t_{2}\left\langle H_{I}\left(\frac{t_{1}}{\lambda^{2}}\right) H_{I}\left(\frac{t_{2}}{\lambda^{2}}\right)\right\rangle_{\varphi_{0}^{(\xi)}},
$$

for $\lambda \rightarrow 0$. Under some regularity conditions on the functions which are used to smear out the (typically) bosonic fields of the reservoir, this limit is shown to exist for many relevant physical models, see [1], and [3, 4, 6] for few recent applications to quantum many body theory. It is at this stage that all the complex quantities like the $\Gamma_{\alpha}^{(\gamma)}$ 's we have introduced in the main body of this paper appear. We define $I(t)=\lim _{\lambda \rightarrow 0} I_{\lambda}(t)$. In the same sense of the convergence of the (rescaled) wave operator $U_{\lambda}\left(\frac{t}{\lambda^{2}}\right)$ (the convergence in the sense of correlators), it is possible to check that also the (rescaled) reservoir operators converge and define new operators which 
do not satisfy canonical commutation relations but a modified version of these. For instance, in Section II this procedure has produced the operators $b_{\alpha, \beta}(t)$ starting from $b(\vec{k})$. Moreover, these limiting operators depend explicitly on time and they live in a Hilbert space which is different from the original one. In particular, they annihilate a vacuum vector, $\eta_{0}$, which is no longer the original one, $\varphi_{0}$. This is what happens, for instance, if $\varphi_{0}$ depends on $\lambda, \varphi_{0} \rightarrow \varphi_{0}^{(\lambda)}$, and considering $\eta_{0}$ as the following limit: $\eta_{0}=\lim _{\lambda \rightarrow 0} \varphi_{0}^{(\lambda)}$.

It is not difficult to deduce the form of a time dependent self-adjoint operator $H_{I}^{(s l)}(t)$, which depends on the system operators and on the limiting operators of the reservoir, such that the first non trivial order of the mean value of the expansion of $U_{t}=\mathbb{1}-i \int_{0}^{t} H_{I}^{(s l)}\left(t^{\prime}\right) U_{t^{\prime}} d t^{\prime}$ on the state $\eta_{0}^{(\xi)}=\eta_{0} \otimes \xi$ coincides with $I(t)$. The operator $U_{t}$ defined by this integral equation is called again the wave operator.

The form of the generator follows now from an operation of normal ordering. More in details, we start defining the flux of an observable $\tilde{X}=X \otimes \mathbb{1}_{r}$, where $\mathbb{1}_{r}$ is the identity of the reservoir and $X$ is an observable of the system, as $j_{t}(\tilde{X})=U_{t}^{\dagger} \tilde{X} U_{t}$. Then, using the equation of motion for $U_{t}$ and $U_{t}^{\dagger}$, we find that $\partial_{t} j_{t}(\tilde{X})=i U_{t}^{\dagger}\left[H_{I}^{(s l)}(t), \tilde{X}\right] U_{t}$. In order to compute the mean value of this equation on the state $\eta_{0}^{(\xi)}$, so to get rid of the reservoir operators, it is convenient to compute first the commutation relations between $U_{t}$ and the limiting operators of the reservoir. At this stage the so called time consecutive principle is used in a very heavy way to simplify the computation. This principle, which has been checked for many classes of physical models, [1], states that, if $\beta(t)$ is any of these limiting operators of the reservoir, then

$$
\left[\beta(t), U_{t^{\prime}}\right]=0, \text { for all } t>t^{\prime} .
$$

Using this principle and recalling that $\eta_{0}$ is annihilated by the limiting annihilation operators of the reservoir, it is now a simple exercise to compute $\left\langle\partial_{t} j_{t}(X)\right\rangle_{\eta_{0}^{(\xi)}}$ and, by means of the equation $\left\langle\partial_{t} j_{t}(X)\right\rangle_{\eta_{0}^{(\xi)}}=\left\langle j_{t}(L(X))\right\rangle_{\eta_{0}^{(\xi)}}$, to identify the form of the generator of the physical system.

Let us now consider the case in which $T>0$. In this case the state of the reservoir is no longer given by the vacuum $\varphi_{0}$. It is now convenient to use the so-called canonical representation of thermal states, [1]. Using the same notation of Section IV, any annihilator operator $a_{\vec{p}, j}$ can be written as the following linear combination

$$
a_{\vec{p}, j}=\sqrt{m(\vec{p})} c_{\vec{p}, j}^{(a)}+\sqrt{n(\vec{p})} c_{\vec{p}, j}^{(b), \dagger},
$$

where $m(\vec{p})$ and $n(\vec{p})$ are the following two-points functions,

$$
m(\vec{p})=\omega_{\beta}\left(a_{\vec{p}, j} a_{\vec{p}, j}^{\dagger}\right)=\frac{1}{1-e^{-\beta \epsilon_{\vec{p}}}}, \quad n(\vec{p})=\omega_{\beta}\left(a_{\vec{p}, j}^{\dagger} a_{\vec{p}, j}\right)=\frac{e^{-\beta \epsilon_{\vec{p}}}}{1-e^{-\beta \epsilon_{\vec{p}}}},
$$


for our bosonic reservoir, if $\omega_{\beta}$ is a KMS state corresponding to an inverse temperature $\beta$. The operators $c_{\vec{p}, j}^{(\alpha)}$ are assumed to satisfy the following commutation rules

$$
\left[c_{\vec{p}, j}^{(\alpha)}, c_{\vec{q}, k}^{(\gamma)}\right]=\delta_{j k} \delta_{\vec{p} \vec{q}} \delta_{\alpha \gamma},
$$

while all the other commutators are trivial. Let moreover $\Phi_{0}$ be the vacuum of the operators $c_{\vec{p}, j}^{(\alpha)}$ :

$$
c_{\vec{p}, j}^{(\alpha)} \Phi_{0}=0, \quad \forall \vec{p}, j, \alpha .
$$

Then it is immediate to check that the results in (A.7) for the KMS state can be found, using these new variables, representing $\omega_{\beta}$ as the following vector state $\omega_{\beta}(\cdot)=\left\langle\Phi_{0}, \cdot \Phi_{0}\right\rangle$. With this GNS-like representation it is trivial to check that both the CCR and the two-point functions are easily recovered. This representation is also called in [1] the Fock-anti Fock representation because of the different sign in the free time evolution of the annihilation operators $c_{\vec{p}, j}^{(a)}$ and $c_{\vec{p}, j}^{(b)}$. Once this representation is introduced, all the same steps as for the situation with $T=0$ can be repeated, and the expression for the generator can be deduced using exactly the same strategy. 


\section{References}

[1] L. Accardi, Y.G. Lu, I. Volovich, Quantum Theory and its Stochastic Limit, Springer, Berlin (2002)

[2] Accardi L., Frigerio A., Lu Y.G., Comm. Math. Phys. 131, 537 (1990) Accardi L., Lu Y.G., Comm. Math. Phys. 180, 605 (1990)

[3] L. Accardi, F. Bagarello, The stochastic limit of the Fröhlich Hamiltonian: relations with the quantum Hall effect, Int. Jour. Theor. Phys., 42, No.10, 2515 (2003)

[4] F. Bagarello, Relations between the Hepp-Lieb and the Alli-Sewell laser models, Ann. H. Poincarè, 3, 983 (2002)

[5] E. Buffet, P.A. Martin, Dynamics of the Open BCS Model, J. Stat. Phys., 18, No. 6, 585 (1978)

[6] F. Bagarello The stochastic limit in the analysis of the open BCS model, J. Phys. A, 37, 2537 (2004)

[7] F. Bagarello The role of a second reservoir in the open BCS model, submitted to IDAQP

[8] F. Bagarello, G. Marchio, F. Strocchi, Quantum Corrections to the Wigner Crystal. An Hartree-Fock Expansion, Phys. Rev. B, 48, 5306 (1993)

[9] T. Chakraborty, P. Pietiläinen, The FQHE, Springer-Verlag, Berlin, 1988.

[10] S.M. Girvin, The Quantum Hall Effect: Novel Excitations and Broken Symmetries, Springer Verlag (1999).

[11] F. Strocchi, Elements of quantum mechanics of infinite systems, World Scientific, Singapore-Philadelphia (1985)

[12] G. Alli and G. L. Sewell, New methods and structures in the theory of the multi-mode Dicke laser model, J. Math. Phys. 36, 5598 (1995)

[13] F. Bagarello, G.L. Sewell, New Structures in the Theory of the Laser Model II: Microscopic Dynamics and a Non-Equilibrim Entropy Principle, J. Math. Phys., 39, 2730 (1998)

[14] R.H. Dicke, Coherence in Spontaneous Radiation Processes Phys. Rev. 93, 99 (1954) 
[15] R. Graham and H. Haken, Laserlight- First example of a second order phase transition far away from thermal equilibrium, Z. Phys. 237, 31 (1970); and H. Haken: Handbuch der Physik, Bd. XXV/2C, Springer, Heidelberg, Berlin, New York, 1970

[16] K. Hepp and E. H. Lieb, Phase transitions in reservoir-driven open systes with applications to lasers and superconductors, Helv. Phys. Acta 46, 573 (1973)

[17] P.A. Martin, Modèles en Mécanique Statistique des Processus Irréversibles, Lecture Notes in Physics, 103, Springer-Verlag, Berlin, (1979)

[18] F. Bagarello, Applications of Topological *-Algebras of Unbounded Operators, J. Math. Phys., 39, 2730 (1998)

[19] F. Bagarello, G. Morchio, Dynamics of mean field spin models from basic results in abstract differential equations, J. Stat. Phys. 66, 849 (1992) 